Article

\title{
Thermal [4 + 2] Cycloadditions of 3-Acetyl-, 3-Carbamoyl-, and 3-Ethoxycarbonyl-Coumarins with 2,3-Dimethyl-1,3-butadiene under Solventless Conditions: A Structural Study
}

\author{
Irma Y. Flores-Larios ${ }^{1}$, Lizbeth López-Garrido ${ }^{2}$, Francisco J. Martínez-Martínez ${ }^{1, *}$, \\ Jorge González ${ }^{1}$, Efrén V. García-Báez ${ }^{2}$, Alejandro Cruz ${ }^{2}$ and Itzia I. Padilla-Martínez ${ }^{2, *}$ \\ 1 Facultad de Ciencias Químicas, Universidad de Colima, km 9 Carretera Coquimatlán-Colima, \\ Coquimatlán Colima 28400, Mexico \\ 2 Departamento de Química, Unidad Profesional Interdisciplinaria de Biotecnología del Instituto \\ Politécnico Nacional, Av. Acueducto s/n, Barrio la Laguna Ticomán 07340, México D. F., Mexico
}

* Authors to whom correspondence should be addressed; E-Mail: ipadillamar@ipn.mx (I.I.P.-M.); fjmartin@ucol.mx (F.J.M.-M.)

Received: 4 December 2009; in revised form: 27 February 2010 / Accepted: 8 March 2010 / Published: 9 March 2010

\begin{abstract}
The thermal [4+2] cycloadditions of 3-acetyl-, 3-carbamoyl, and 3-ethoxycarbonylcoumarins with 2,3-dimethyl-1,3-butadiene under solvent free conditions are reported, as well as the epoxidation reactions of some adducts. Discussion is focused on the structural features of the Diels-Alder adducts and their epoxides, based upon NMR, $\mathrm{X}$-ray, and mass spectral data, and supported by ab initio theoretical calculations.
\end{abstract}

Keywords: coumarins; Diels-Alder adducts; solventless reactions; 6a,7,7a,8a,9,9ahexahydro-7a,8a-dimethyl-6-oxo-6H-5,8-dioxacyclopropa[b]phenantrenes

\section{Introduction}

Coumarins are widely known to undergo pericyclic reactions like photodimerization [1]. On this topic and in the context of crystal engineering, our group has reported the solid state photodimerization of ethyl coumarin-3-carboxylate (1a) and its 6-Cl and 6-Br derivatives $\mathbf{1 b}$ and $\mathbf{1 c}$ [2]. In contrast, the use of coumarins as $2 \pi$ components in Diels-Alder (DA) cycloadditions has been less studied due to 
their low reactivity in these reactions. Only 3-substituted coumarins with electron-withdrawing groups like COOEt [3], $\mathrm{NO}_{2}$ [4], $\mathrm{SO}_{2} \mathrm{Ph}$, or heterocyclic rings [5], and very recently with $\mathrm{CN}$ [6], have been reported to undergo DA reactions under high pressure conditions.

The DA reaction of 1a with 2,3-dimethyl-1,3-butadiene was realized in water alone and in $\mathrm{CH}_{2} \mathrm{Cl}_{2}$ under $9 \mathrm{kbar}$ pressure [5]. The use of $\mathrm{HfCl}_{4} \cdot 2 \mathrm{THF}$ as catalyst, and solvent free conditions (SFC) significantly improve selectivity and yields [7]. However, independent results, obtained from our group, indicate that this reaction can be performed without catalyst under SFC [8]. Thus, in this work the synthesis of the $(6 \mathrm{a} R, 10 \mathrm{a} R)$-rel-6a,7,10,10a-tetrahydro-8,9-dimethyl-6-oxodibenzo[b, $d]$ pyran derivatives 6-10 from the DA reaction of 3-acetyl-, 3-carbamoyl-, and 3-ethoxycarbonyl-coumarins 15 with 2,3-dimethyl-1,3-butadiene under SFC as well as the synthesis of some of their epoxides 11-15 is reported (Scheme 1). The molecular structure of the compounds obtained is discussed on the basis of their NMR and X-ray data.

Scheme 1. Synthesis scheme and numbering.

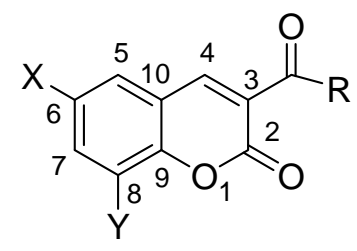

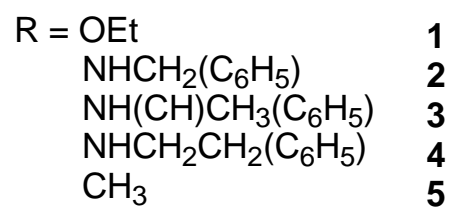

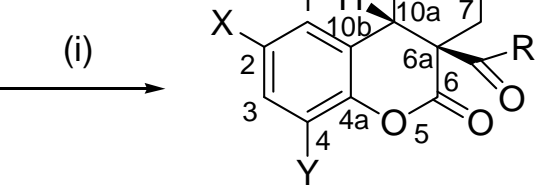

6

7

8

9

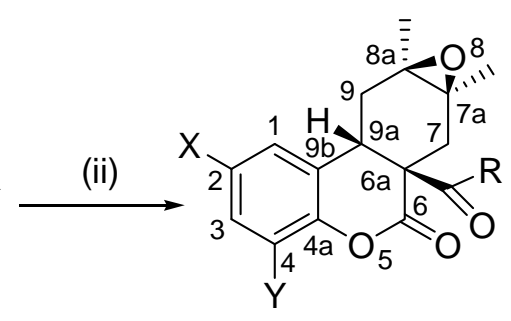

11

12

13

14

15

$$
\begin{array}{llllllllll} 
& \mathbf{a} & \mathbf{b} & \mathbf{c} & \mathbf{d} & \mathbf{e} & \mathbf{f} & \mathbf{g} & \mathbf{h} & \mathbf{i} \\
\mathrm{X}= & \mathrm{H} & \mathrm{Cl} & \mathrm{Br} & \mathrm{NO}_{2} & \mathrm{OMe} & \mathrm{H} & \mathrm{Cl} & \mathrm{Cl} & \mathrm{Br} \\
\mathrm{Y}= & \mathrm{H} & \mathrm{H} & \mathrm{H} & \mathrm{H} & \mathrm{H} & \mathrm{OMe} & \mathrm{Cl} & \mathrm{Br} & \mathrm{OMe}
\end{array}
$$

(i) 2,3-Dimethyl-1,3- butadiene under SFC; (ii) $m$-Chloroperbenzoic acid in $\mathrm{CHCl}_{3}$

\section{Results and Discussion}

The synthesis of the cycloadducts was performed, starting from coumarins 1-5, in a sealed glass ampoule with an excess of the diene (6 equivalents) at $160{ }^{\circ} \mathrm{C}$, to give the corresponding DA cycloadducts 6-10, in moderate to good yields (60-85\%). The isolated yield of the adduct $\mathbf{6 a}(80 \%)$, after purification by column chromatography, is lower than that reported in $\mathrm{CH}_{2} \mathrm{Cl}_{2}$ at high pressure [5] or under SFC in the presence of catalysts [7], but higher than that reported in water at $150{ }^{\circ} \mathrm{C}(58 \%)$ [5]. The cycloadduct $7 \mathbf{a}$ was obtained in $85 \%$ yield, which is higher than the reported value (76\%) using toluene as solvent [7].

In all cases racemic mixtures of the cis fused rings were formed, except in the case of $\mathbf{8 a}$ which was synthesized using enantiopure $(R)$-1-phenylethylamine to generate coumarin 3a. Thus, it is assumed that 8a was obtained as a $60: 40$ mixture of $\left(6 \mathrm{a} S, 10 \mathrm{a} S, 1^{\prime} R\right)$ and $\left(6 \mathrm{a} R, 10 \mathrm{a} R, 1^{\prime} R\right)$ diastereomers, respectively. For this mixture, two sets of signals in the ${ }^{1}$ H-NMR spectrum are clearly observed at $\delta$ 
5.89, 1.21 (major) and 5.82, 1.37 (minor). They are doublets assigned to the $\mathrm{NH}$ and $\mathrm{CH}_{3}$ protons of the amide moiety, respectively. In the former set, the signal for the $\mathrm{CH}_{3}$ protons appears shielded because of the effect exerted by the coumarin aromatic ring diamagnetic currents. The ab initio calculated molecular geometry of $\left(6 \mathrm{a} S, 10 \mathrm{a} S, 1^{\prime} R\right)$ and $\left(6 \mathrm{a} R, 10 \mathrm{a} R, 1^{\prime} \mathrm{R}\right)$ diastereomers predicts that the $\mathrm{CH}_{3}$ protons, in the former, are in the appropriate position to be shielded by diamagnetic currents of the aromatic ring, with $1.24 \mathrm{kcal} \mathrm{mol}^{-1}$ in favour of the $\left(6 \mathrm{aS}, 10 \mathrm{a} S, 1^{\prime} R\right)$ diatereomer. These results are in agreement with the preference of the diene approach to the less hindered face of the starting coumarin 3a (Figure 1). However, the asymmetric induction of the chiral amine pendant group is poor in comparison with the results obtained for bulkier 3-alkoxides [10], because of its relatively long distance from the reactive double bond.

Figure 1. Calculated molecular structures of the $\left(6 \mathrm{a} S, 10 \mathrm{a} S, 1^{\prime} R\right)$ and $\left(6 \mathrm{a} R, 10 \mathrm{a} R, 1^{\prime} R\right)$ diastereomers of the cycloadduct 8a. In the former (left) the methyl protons of the chiral amine residue lie in the shielding cone of the coumarin benzenoid ring.

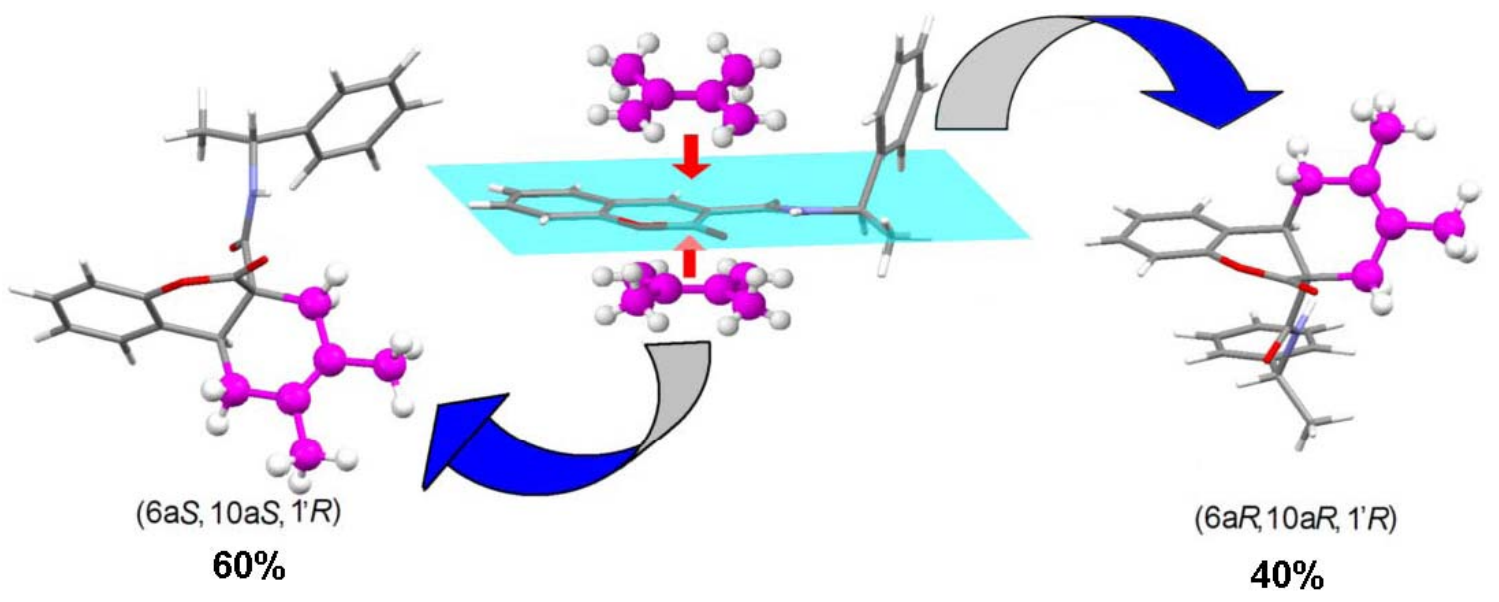

In order to test the stereofacial selectivity of the addition reaction on the cyclohexene ring, the epoxidation of compounds $\mathbf{6 a - 1 0 a}$ and 10d,f,i with $m$-chloroperbenzoic acid ( $m$-CPBA) was performed. The reaction proceeded in moderate $70-80 \%$ (11a-15a) to very good yields $90-96 \%$ (15d,f,i). The X-ray data (vide infra) show that the oxygen atom is stereoselectively added to the less hindered face of the cyclohexene ring, opposite to the benzopyrone ring. Therefore, the racemic mixture $(6 \mathrm{a} R, 7 \mathrm{a} R, 8 \mathrm{a} S, 9 \mathrm{a} R)$ and $(6 \mathrm{a} S, 7 \mathrm{a} S, 8 \mathrm{a} R, 9 \mathrm{a} S)$ is formed except in the case of compound 13a, which was obtained as a 60:40 mixture of diastereomers because of the presence of the amine moiety stereocentre. Thus, the original diastereomeric ratio of the starting adduct 8a is preserved (vide supra).

The molecular structure in solution was analyzed by ${ }^{1} \mathrm{H}$ - and ${ }^{13} \mathrm{C}-\mathrm{NMR}$, the numbering scheme is given in Figure 1. Several differences in the ${ }^{1} \mathrm{H}-\mathrm{NMR}$ spectra appear as a consequence of the cycloaddition. The H-4 signal in coumarins 1-5 usually appears as a singlet between 8.0 and $8.4 \mathrm{ppm}$ [11], whereas in the cycloadducts 6-10 it becomes H-10a and appears as a doublet of doublets, by coupling with $\mathrm{H}_{2}-10$, in the range 3.36-3.65 ppm. Irradiation of $\mathrm{H}-10$ a signal gave NOEs with $\mathrm{H}-1$, $\mathrm{H}_{\mathrm{eq}}-10$, and alkyl protons of the $\mathrm{R}$ group, confirming the cis fusion between dihydropyrone and cyclohexene rings. Besides NOE experiments, the assignments of all ${ }^{1} \mathrm{H}$ signals were achieved through COSY experiments. The mean values of the coupling constants of $\mathrm{H}-10 \mathrm{a}$ with $\mathrm{H}_{\mathrm{ax}}-10(11.0-12.6 \mathrm{~Hz})$ 
and $\mathrm{H}_{\mathrm{eq}}-10(5.0-6.5 \mathrm{~Hz})$, suggest a pseudo axial-axial and pseudo axial-equatorial relationship, respectively, and thus an anchored conformation for the cyclohexene ring. The nature of the carbonyl group at the 6a position exerts influence on the chemical shift of H-10a: for COOEt and COMe, H-10a appears in the range of 3.36-3.47 ppm whereas for CONHR it appears more deshielded, in the range 3.58-3.65 ppm, due to the effect of the amide mesomerism. The chemical shift of $\mathrm{H}_{\mathrm{ax}}-7$ in the acetylated adducts $\mathbf{1 0}$ appears at higher field $(2.04-2.38 \mathrm{ppm})$ than in the carbamoyl and ethoxycarbonyl adducts 6a-8a (2.47-2.50 ppm). This trend could be explained by a syn or anti conformational preference of the 3-CO with respect to the lactone carbonyl moiety. In compounds 6a8a the most populated conformer on the ${ }^{1} \mathrm{H}$ - NMR time scale is the anti one with the 3-CO group appropriately positioned to exert a deshielding effect on $\mathrm{H}_{\mathrm{ax}}-7$, whereas the syn conformer is the predominant form in adducts 10a-i. The chemical shift of $\mathrm{H}_{\mathrm{ax}}-7$, in the adduct 9a, is out of range $(\delta 2.30)$ because of the protective effect exerted by the phenyl ring of the 2-phenylethyl amine residue. Finally, the chemical shift of $\mathrm{H}_{\mathrm{eq}}-7$ is in the range of 2.78 to $2.91 \mathrm{ppm}$, due to the deshielding effect of the dihydropyrone carbonyl moiety.

The change in the hybridization of C-3 and C-4 from $s p^{2}$ in coumarins 1-5 to $s p^{3}$ character in adducts 6-10 shifts the corresponding carbon atoms C-6a and C-10a, to lower frequencies, from 118125 to 54-61 and from 147-149 to 36-37 ppm, respectively. The nature of the 3-substituent influences the chemical shift of the carbon atom carrying the substituent: thus C-6a appears in the range of 54-55 ppm for amide and ester adducts 6a-9a and at 60-61 ppm for the acetylated adducts 10a-i. The difference in chemical shifts is preserved from the starting coumarins: C-3 resonates at 118-120 ppm in compounds $\mathbf{1} \mathbf{a}-\mathbf{4} \mathbf{a}^{9}$ and at $124-125$ ppm in $\mathbf{5 a - i}$.

Epoxidation changes the hybridation of C-8 and C-9 atoms from $s p^{2}$ in the cycloadducts 6-10 to $s p^{3}$ character in epoxides 11a-15a and 15d,f,i, shifting the corresponding C-7a and C-8a carbon atoms approximately by $62 \mathrm{ppm}$ to lower frequencies. Subtle changes are also observed in the ${ }^{1} \mathrm{H}-\mathrm{NMR}$ spectra: the oxirane methyl protons, $\mathrm{H}_{\mathrm{ax}}-7$, and $\mathrm{H}_{\mathrm{ax}}-9$ (these last $\mathrm{H}_{\mathrm{ax}}-10$ before the epoxidation) are shifted to low frequencies by approximately $0.3 \mathrm{ppm}$, owing to the effect of the steric compression exerted by the new formed three-membered ring.

Figure 2. Molecular structure of cycloadduct $\mathbf{1 0 b}$ and $\mathbf{1 5 i}$. Thermal ellipsoids drawn at the $50 \%$ probability level.

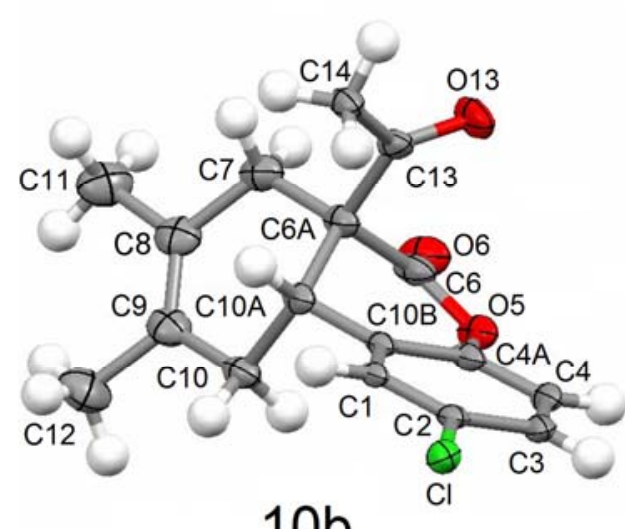

$10 \mathrm{~b}$

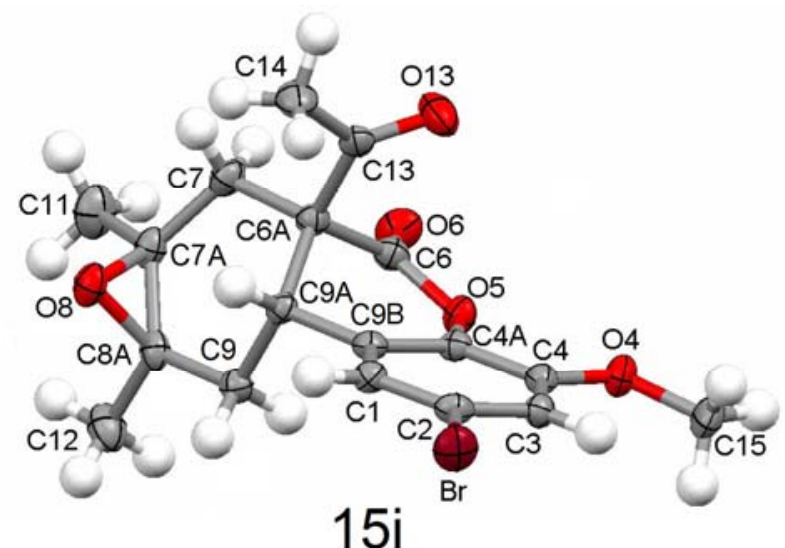

$15 i$ 
The molecular structures of cycloadduct $\mathbf{1 0 b}$ and epoxide $\mathbf{1 5 i}$, obtained by X-ray diffraction, are shown in Figure 2. Selected bond lengths and angles are listed in Table 1. In consequence of the transformation of $\mathrm{C} 3-\mathrm{C} 4$ double bond in coumarins to the single bond $\mathrm{C} 6 \mathrm{a}-\mathrm{C} 10 \mathrm{a}$ in the adducts, this bond length enlarges by $0.18(2) \AA$, from 1.359(2) in 5a [12] to 1.538(3) in 10b. Epoxidation of adducts also changes the hybridation of $\mathrm{C} 8$ and $\mathrm{C} 9$ atoms enlarging $\mathrm{C} 8-\mathrm{C} 9$ bond length by $0.13(1) \AA$, in agreement with their new $s p^{3}$ character, from $1.331(3)$ in $\mathbf{1 0 b}$ to $1.462(7)$ in $15 \mathbf{i}(\mathrm{C} 7 \mathrm{~A}-\mathrm{C} 8 \mathrm{~A})$, respectively.

Table 1. Selected bond lengths and angles from X-ray data of compounds $\mathbf{1 0 b}$ and $\mathbf{1 5 i}$.

\begin{tabular}{|c|c|c|}
\hline Atoms & $10 b(X=C l)$ & $15 i(X=B r)$ \\
\hline \multicolumn{3}{|c|}{ Bond lengths/ $\AA$} \\
\hline $\mathrm{O}(5) \mathrm{C}(6)$ & $1.373(3)$ & $1.364(6)$ \\
\hline $\mathrm{O}(6) \mathrm{C}(6)$ & $1.190(3)$ & $1.195(6)$ \\
\hline$C(6) C(6 A)$ & $1.526(3)$ & $1.522(7)$ \\
\hline$C(6 \mathrm{~A}) \mathrm{C}(7)$ & $1.536(3)$ & $1.541(6)$ \\
\hline$C(6 A) C(10 A)$ & $1.538(3)$ & $1.551(6)$ \\
\hline $\mathrm{C}(6 \mathrm{~A}) \mathrm{C}(13)$ & $1.537(3)$ & $1.527(6)$ \\
\hline $\mathrm{C}(7) \mathrm{C}(8)$ & $1.509(3)$ & \\
\hline $\mathrm{C}(7) \mathrm{C}(7 \mathrm{~A})$ & & $1.501(7)$ \\
\hline $\mathrm{C}(8) \mathrm{C}(9)$ & $1.331(3)$ & \\
\hline $\mathrm{C}(7 \mathrm{~A}) \mathrm{C}(8 \mathrm{~A})$ & & $1.462(7)$ \\
\hline $\mathrm{C}(9) \mathrm{C}(10)$ & $1.500(3)$ & \\
\hline $\mathrm{C}(8 \mathrm{~A}) \mathrm{C}(9)$ & & $1.505(6)$ \\
\hline$C(10) C(10 A)$ & $1.530(3)$ & \\
\hline $\mathrm{C}(9) \mathrm{C}(9 \mathrm{~A})$ & & $1.523(6)$ \\
\hline $\mathrm{XC}(2)^{\mathrm{a}}$ & $1.742(2)$ & $1.900(5)$ \\
\hline \multicolumn{3}{|c|}{ Bond angles/ ${ }^{\circ}$} \\
\hline $\mathrm{C}(4 \mathrm{~A}) \mathrm{O}(5) \mathrm{C}(6)$ & $120.94(16)$ & $120.2(4)$ \\
\hline $\mathrm{C}(6 \mathrm{~A}) \mathrm{C}(7) \mathrm{C}(8)$ & $115.64(18)$ & $117.2(4)$ \\
\hline$C(9) C(10) C(10 A)$ & $112.61(18)$ & \\
\hline $\mathrm{C}(8 \mathrm{~A}) \mathrm{C}(9) \mathrm{C}(9 \mathrm{~A})$ & & $113.7(4)$ \\
\hline $\mathrm{O}(13) \mathrm{C}(13) \mathrm{C}(6 \mathrm{~A})$ & $120.30(19)$ & $121.1(5)$ \\
\hline $\mathrm{XC}(2) \mathrm{C}(1)^{\mathrm{a}}$ & $119.68(15)$ & $119.1(4)$ \\
\hline \multicolumn{3}{|c|}{ Torsion angles/ ${ }^{\circ}$} \\
\hline $\mathrm{C}(6) \mathrm{O}(5) \mathrm{C}(4 \mathrm{~A}) \mathrm{C}(10 \mathrm{~B})$ & $20.4(3)$ & $23.2(6)$ \\
\hline $\mathrm{C}(7) \mathrm{C}(6 \mathrm{~A}) \mathrm{C}(6) \mathrm{O}(5)$ & $166.50(17)$ & $163.0(4)$ \\
\hline $\mathrm{O}(5) \mathrm{C}(6) \mathrm{C}(6 \mathrm{~A}) \mathrm{C}(13)$ & $74.9(2)$ & $77.6(5)$ \\
\hline $\mathrm{C}(6) \mathrm{C}(6 \mathrm{~A}) \mathrm{C}(13) \mathrm{O}(13)$ & $15.2(3)$ & $2.6(6)$ \\
\hline $\mathrm{O}(13) \mathrm{C}(13) \mathrm{C}(6 \mathrm{~A}) \mathrm{C}(7)$ & $104.8(2)$ & $116.7(5)$ \\
\hline $\mathrm{O}(8) \mathrm{C}(9) \mathrm{C}(10) \mathrm{C}(10 \mathrm{~A})$ & $23.4(3)$ & \\
\hline $\mathrm{O}(8) \mathrm{C}(8 \mathrm{~A}) \mathrm{C}(9) \mathrm{C}(9 \mathrm{~A})$ & & $-42.6(5)$ \\
\hline $\mathrm{C}(10 \mathrm{~A}) \mathrm{C}(10 \mathrm{~B}) \mathrm{C}(4 \mathrm{~A}) \mathrm{O}(5)$ & $-3.2(3)$ & \\
\hline $\mathrm{C}(9 \mathrm{~A}) \mathrm{C}(9 \mathrm{~B}) \mathrm{C}(4 \mathrm{~A}) \mathrm{O}(5)$ & & $-1.6(7)$ \\
\hline $\mathrm{C}(7) \mathrm{C}(8) \mathrm{C}(9) \mathrm{C}(10)$ & $0.4(3)$ & \\
\hline $\mathrm{C}(7) \mathrm{C}(7 \mathrm{~A}) \mathrm{C}(8 \mathrm{~A}) \mathrm{C}(9)$ & & $0.9(7)$ \\
\hline $\mathrm{C}(9 \mathrm{~B}) \mathrm{C}(1) \mathrm{C}(2) \mathrm{X}$ & $179.04(13)$ & $177.9(3)$ \\
\hline
\end{tabular}


The X-ray structures of compounds $\mathbf{1 0 b}$ and $\mathbf{1 5 i}$ show that the $\mathrm{CO}$ of the acetyl group is pointing towards the lactone ring $\left[\mathrm{C}(7) \mathrm{C}(6 \mathrm{~A}) \mathrm{C}(13) \mathrm{O}(13)\right.$ torsion angles of $-104.8(2)$, -and $-116.7(5)^{\mathbf{o}}$ for $\mathbf{1 0 b}$ and 15i, respectively. Thus, the syn conformation between both carbonyls is the preferred in the solid state, the same conformational preference being observed in solution by NMR (vide supra). The torsion angles $\mathrm{C}(10 \mathrm{~A}) \mathrm{C}(10 \mathrm{~B}) \mathrm{C}(4 \mathrm{~A}) \mathrm{O}(5)(\mathbf{1 0 b})$ and $\mathrm{C}(9 \mathrm{~A}) \mathrm{C}(9 \mathrm{~B}) \mathrm{C}(4 \mathrm{~A}) \mathrm{O}(5)$ (15i) in dihydropyrone ring, and $\mathrm{C}(7) \mathrm{C}(8) \mathrm{C}(9) \mathrm{C}(10)(\mathbf{1 0 b})$ and $\mathrm{C}(7) \mathrm{C}(7 \mathrm{~A}) \mathrm{C}(8 \mathrm{~A}) \mathrm{C}(9)(\mathbf{1 5 i})$ in cyclohexene ring, take values near to zero, in agreement with a distorted twisted boat conformation for both rings. Epoxydation has a negligible influence on the conformation of the cyclohexane ring as observed in compound $\mathbf{1 5 i}$.

The supramolecular structure of DA adduct $\mathbf{1 0 b}$ and epoxide $\mathbf{1 5} \mathbf{i}$ is organized by weak $\mathrm{CH} \cdots \mathrm{A}$ $(\mathrm{A}=\mathrm{O}, \pi)$ interactions. Nevertheless, they are scarce in comparison with those encountered in the crystal packing of the starting coumarins [12]. Thus, compound 15i is worthy to mention, since its crystal network is organized by several $\mathrm{CH}^{\cdots} \mathrm{O}$ and $\mathrm{Br} \cdots \mathrm{Br}$ polarization-induced interactions, in the $b c$ plane [Figure 3(a)], and $\mathrm{CH}^{\cdots} \pi$ bifacial contacts developing the third dimension along the $\left(\begin{array}{lll}7 & 0 & 3\end{array}\right)$ direction [(Figure 3(b)]. The $\mathrm{Br}^{\cdots} \mathrm{Br}^{i}$ distance of 3.496(6) $\AA$ (symmetry code: (i) 1-x, 1-y, 2-z) is shorter than the reported value of 3.618(4) $\AA$ for 6-bromo- $N$-(2-hydroxyethyl)-2-oxo-2H-1benzopyran-3-carboxamide [13]. The geometric parameters associated with non covalent interactions are listed in Table 2, $\mathrm{C}-\mathrm{H} \cdots \mathrm{O}[14]$ and $\mathrm{C}-\mathrm{H} \cdots \pi[15]$ interactions are in agreement with accepted criteria and particularly with values reported for other coumarins $[12,16]$.

Figure 3. Supramolecular structure of compound 15i. (a) View in the $b c$ plane showing $\mathrm{CH} \cdots \mathrm{O}$ and $\mathrm{Br} \cdots \mathrm{Br}$ contacts. (b) View of bifacial $\mathrm{CH}^{\cdots} \cdot \pi$ contacts developing the third dimension along the $\left(\begin{array}{lll}7 & 0 & 3\end{array}\right)$ direction. Dashed lines represents intermolecular $\mathrm{CH} \cdots \mathrm{A}$ $(\mathrm{A}=\mathrm{O}, \pi)$ contacts.

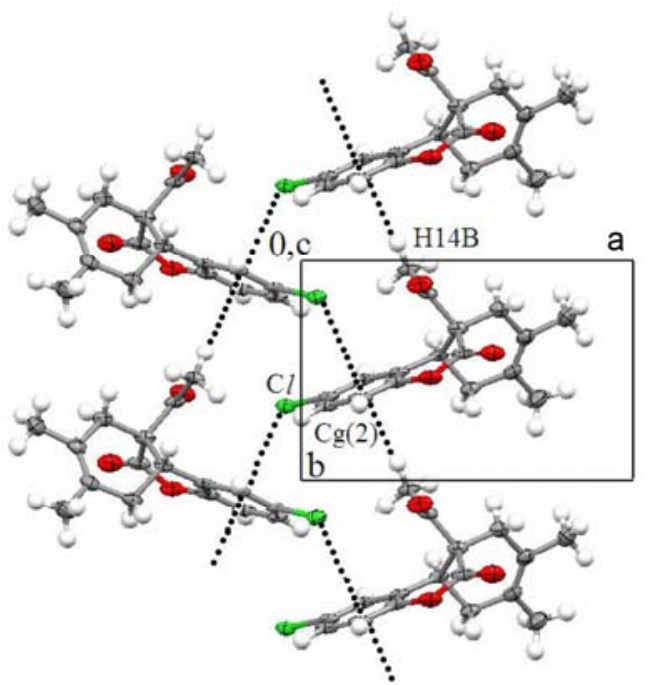

(a)

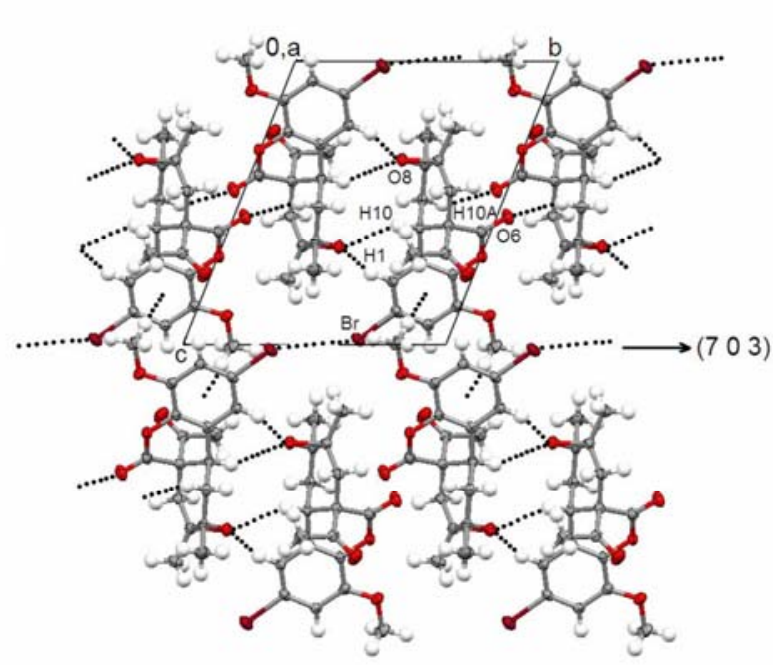

(b) 
Table 2. Geometric parameters associated with intermolecular hydrogen interactions in compounds $\mathbf{1 0 b}$ and $\mathbf{1 5 i}$.

\begin{tabular}{|c|c|c|c|c|c|}
\hline Compound & D-H $\cdots$ A (symmetry code) & $\mathrm{D}-\mathbf{H} / \AA$ & $\mathbf{H} \cdots \mathbf{A} / \AA$ & $\mathbf{D} \cdots \mathbf{A} / \AA$ & $\mathbf{D}-\mathbf{H} \cdots \mathbf{A} /^{\circ}$ \\
\hline \multirow[t]{2}{*}{$10 \mathrm{~b}$} & $\mathrm{C}(14)-\mathrm{H} 14 \mathrm{~B} \cdots \operatorname{Cg}(2)(\mathrm{x}, \mathrm{y}+1, \mathrm{z})$ & & 2.66 & $3.626(2)$ & 169 \\
\hline & $\mathrm{C}(10)-\mathrm{H}(10) \cdots \mathrm{O}(13)(\mathrm{x}, 1 / 2-\mathrm{y}, \mathrm{z}+1 / 2)$ & & 2.37 & $3.199(2)$ & 140 \\
\hline \multirow[t]{6}{*}{$15 \mathbf{i}$} & $\mathrm{C}(1)-\mathrm{H}(1) \cdots \mathrm{O}(8)(-\mathrm{x}, 1-\mathrm{y}, 1-\mathrm{z})$ & 0.93 & 2.56 & $3.317(6)$ & 139 \\
\hline & $\mathrm{C}(9)-\mathrm{H}(10 \mathrm{~A}) \cdots \mathrm{O}(8)(-\mathrm{x}, 1-\mathrm{y}, 1-\mathrm{z})$ & 0.98 & 2.59 & $3.491(6)$ & 153 \\
\hline & $\mathrm{C}(14)-\mathrm{H}(14 \mathrm{~A}) \cdots \mathrm{O}(8)(-\mathrm{x}, 1-\mathrm{y}, 1-\mathrm{z})$ & 0.96 & 2.51 & $3.421(7)$ & 160 \\
\hline & $\mathrm{C}(9)-\mathrm{H}(9 \mathrm{~A}) \cdots \mathrm{O}(6)(1-\mathrm{x}, 2-\mathrm{y}, 1-\mathrm{z})$ & 0.97 & 2.51 & $3.428(6)$ & 157 \\
\hline & $\mathrm{C}(14)-\mathrm{H}(14 \mathrm{C}) \cdots C g(3)^{\mathrm{a}}(\mathrm{x}-1, \mathrm{y}, \mathrm{z})$ & & 2.76 & $3.603(6)$ & 147 \\
\hline & $\begin{array}{l}\mathrm{C}(15)-\mathrm{H}(15 \mathrm{~A}) \cdots C g(3)^{\mathrm{a}}(1-\mathrm{x}, 2-\mathrm{y}, 2- \\
\mathrm{z})\end{array}$ & & 2.92 & $3.764(6)$ & 148 \\
\hline
\end{tabular}

${ }^{\mathrm{a}} \mathrm{Cg}(3)$ is the centroid of the benzenoid ring $(\mathrm{C} 1-\mathrm{C} 4 / \mathrm{C} 4 \mathrm{a} / \mathrm{C} 9 \mathrm{~B})$.

The molecular peaks of 3-acetylcoumarin adducts 10 and their epoxides 15a,d,f,i are barely observed $(1 \%)$ by mass spectrometry. Nevertheless all compounds are cleaved and rearranged following the typical fragmentation path depicted in Scheme 2. Cycloadducts and epoxides give the corresponding fragments, $m / z 227$ for 6a-10a and 243 for 11a-15a, by the loss of 3-ethoxycarbonyl, 3-carbamoyl, or 3-acetyl angular groups. The species derived from 6-10 are further broken by the loss of CO and rearranged to the 2,3-dimethyl-1,4,4a,9b-tetrahydro-dibenzofuran ion $(\mathrm{m} / z \mathrm{199})$ or by the loss of dimethylacetylene $(\mathrm{m} / \mathrm{z} 54)$ to form the oxacyclobutanaphtalene species $(\mathrm{m} / \mathrm{z} 173)$. The species derived from epoxides 11-15 mainly rearrange to the corresponding dibenzofuran $(\mathrm{m} / \mathrm{z} 225)$ or chromene species $(\mathrm{m} / \mathrm{z} 211)$ after the loss of water or $\mathrm{O}_{2}$, respectively. Both fragmentation paths are in agreement with those found for other coumarins [17]. It is worthy to mention that retro-DA conversion was observed only in compound $\mathbf{1 0 e}$.

Scheme 2. Typical fragmentation path of DA adducts 6a-10ai and epoxides 11a-15a-i by mass spectrometry.

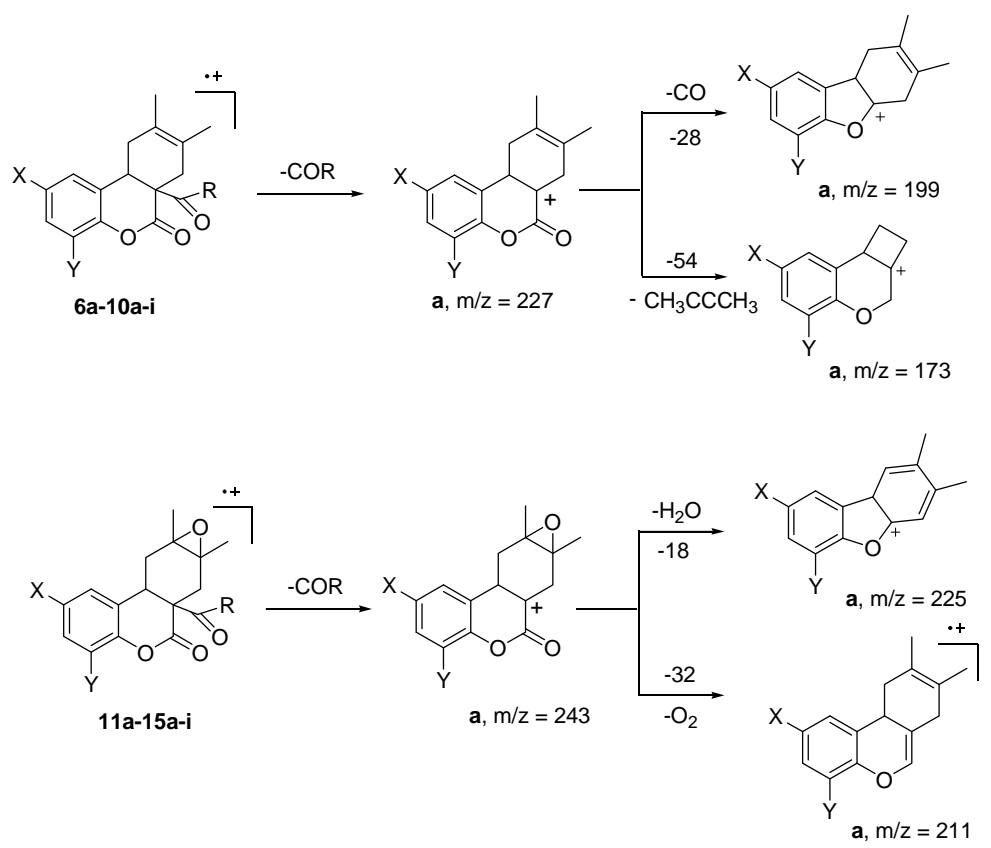




\section{Experimental}

\subsection{General methods}

All chemicals and solvents were of reagent grade and used as received. Melting points were measured on an Electrothermal IA 9100 apparatus and were uncorrected. IR spectra were recorded in $\mathrm{KBr}$ disks using a Perkin-Elmer 16F PC IR spectrophotometer. Mass spectra were obtained in a GC/MS system (Varian) with an electron ionization mode. Elemental analyses (EA) were performed on a Perkin-Elmer 2400 elemental analyzer. ${ }^{1} \mathrm{H}$ - and ${ }^{13} \mathrm{C}-\mathrm{NMR}$ spectra were recorded on a Varian Mercury $300\left({ }^{1} \mathrm{H}, 300.08 ;{ }^{13} \mathrm{C}, 75.46 \mathrm{MHz}\right)$ instrument in $\mathrm{CDCl}_{3}$ solutions, unless otherwise specified, measured with $\mathrm{SiMe}_{4}$ as the internal reference, $\delta$ are in ppm and coupling constants ${ }^{\mathrm{n}} J$ in $\mathrm{Hz}$. ${ }^{1} \mathrm{H}$ - and ${ }^{13} \mathrm{C}-\mathrm{NMR}$ assignments were achieved on the basis of NOE, COSY and HETCOR experiments. Singlecrystal X-ray diffraction data for molecules $\mathbf{1 0 b}$ and 15i were collected on a Bruker Apex II area detector diffractometer at 100 and $293 \mathrm{~K}$, respectively, with Mo $\mathrm{K} \alpha$ radiation, $\lambda=0.71073 \AA$. A semiempirical absorption correction was applied using SADABS [18], and the program SAINT [18] was used for integration of the diffraction profiles. The structures were solved by direct methods using SHELXS97 [19] program of WinGX package [20]. The final refinement was performed by full-matrix least-squares methods on $F^{2}$ with SHELXL97 [19] program. H atoms on C, N and O were positioned geometrically and treated as riding atoms, with $\mathrm{C}-\mathrm{H}=0.93-0.98 \AA$, and with $U$ iso $(\mathrm{H})=1.2 \operatorname{Ueq}(\mathrm{C})$. Mercury was used for visualization, molecular graphics and analysis of crystal structures [21], software used to prepare material for publication was PLATON [22]. Crystallographic data (excluding structure factors) for the structures in this paper have been deposited with the Cambridge Crystallographic Data Centre as supplementary publication CCDC numbers 735605 (10b) and 721872 (15i). Copies of the data can be obtained, free of charge, on application to CCDC, 12 Union Road, Cambridge CB2 1EZ, UK, (Fax: +44-01223-336033 or E-Mail: deposit@ccdc.cam.ac.uk). Crystals suitable for X-ray analysis were obtained from saturated $\mathrm{CHCl}_{3}$ solutions. The program GAUSSIAN98 [23] was used to perform the ab initio molecular orbital calculations at RHF-631G** level of theory.

\subsection{General synthetic procedure for coumarins $\mathbf{1 - 5}$}

The starting coumarins 1a-4a were synthesized according with the methodology reported elsewhere [11,16,24]. 6-Substituted acetyl coumarins 5a-i were synthesized by Knoevenagel condensation of ethyl acetoacetate and the corresponding 5-substituted 2-hydroxybenzaldehyde, the spectroscopic data of 5a-d are in agreement with literature [25].

3-Acetyl-6-methoxy-2H-1-benzopyran-2-one (5e). Prepared from $0.41 \mathrm{~mL}$ (3.3 mmol) of 2-hydroxy-5methoxybenzaldehyde and $0.42 \mathrm{~mL}(3.3 \mathrm{mmol})$ of ethyl acetoacetate. Yellow solid $89 \%$ yield, $\mathrm{mp}$ 180-183 ${ }^{\circ} \mathrm{C}$. IR v $\left(\mathrm{cm}^{-1}\right): 1723(\mathrm{OC}=\mathrm{O}), 1677(\mathrm{C}=\mathrm{O}), 1226,1197(\mathrm{C}-\mathrm{O}) .{ }^{1} \mathrm{H}-\mathrm{NMR}: 8.44$ (s, 1H, H4), $7.28\left(\mathrm{~d}, 1 \mathrm{H},{ }^{3} \mathrm{~J}=9.1, \mathrm{H} 8\right), 7.20\left(\mathrm{dd}, 1 \mathrm{H},{ }^{3} \mathrm{~J}=9.1,{ }^{4} \mathrm{~J}=2.9, \mathrm{H} 7\right), 7.02\left(\mathrm{~d}, 1 \mathrm{H},{ }^{4} \mathrm{~J}=2.6, \mathrm{H} 5\right), 3.85$ (s, 3H, $\left.\mathrm{OCH}_{3}\right), 2.70\left(\mathrm{~s}, 3 \mathrm{H}, \mathrm{CH}_{3}\right) ;{ }^{13} \mathrm{C}-\mathrm{NMR}: 195.9(\mathrm{CO}), 159.7$ (OCO), $156.6(\mathrm{C} 6), 150.1$ (C10), $147.6(\mathrm{C} 4)$, 124.8 (C3), 123.2 (C7), 117.9 (C5), 118.7 (C9), 111.3 (C8), $56.1\left(\mathrm{OCH}_{3}\right), 30.9\left(\mathrm{CH}_{3}\right)$; EA (\%) calculated for $\mathrm{C}_{12} \mathrm{H}_{10} \mathrm{O}_{4}$ : $66.05 \mathrm{C}, 4.62 \mathrm{H}$; found: $66.04 \mathrm{C}, 4.61 \mathrm{H}$. 
3-Acetyl-8-methoxy-2H-1-benzopyran-2-one (5f). Prepared from $0.5 \mathrm{~g}(3.3 \mathrm{mmol})$ of 2-hydroxy-3methoxybenzaldehyde and $0.42 \mathrm{~mL}(3.3 \mathrm{mmol})$ of ethyl acetoacetate. Yellow solid, $88 \%$ yield, mp 171-174 ${ }^{\circ} \mathrm{C}$. IR v(cm $\left.{ }^{-1}\right): 1727(\mathrm{OC}=\mathrm{O}), 1682(\mathrm{C}=\mathrm{O}), 1278,1197(\mathrm{C}-\mathrm{O}) .{ }^{1} \mathrm{H}-\mathrm{NMR}: 8.42$ (s, 1H, H4), $7.25\left(\mathrm{~d}, 1 \mathrm{H},{ }^{4} \mathrm{~J}=1.1,{ }^{3} \mathrm{~J}=5.7, \mathrm{H} 7\right), 7.14\left(\mathrm{~d}, 1 \mathrm{H},{ }^{4} \mathrm{~J}=2.0,{ }^{3} \mathrm{~J}=5.7, \mathrm{H} 5\right), 7.18\left(\mathrm{t}, 1 \mathrm{H},{ }^{3} J=5.5,{ }^{4} \mathrm{~J}=2.0\right.$, H6), 3.94 (s, 3H, $\left.\mathrm{OCH}_{3}\right), 2.68$ (s, 3H, $\left.\mathrm{CH}_{3}\right) ;{ }^{13} \mathrm{C}-\mathrm{NMR}: 195.8$ (CO), 158.9 (OCO), 145.1 (C10), 147.9 (C4), 147.2 (C8), 125.0 (C5), 124.8 (C3), 121.5 (C6), 116.0 (C7), 118.9 (C9), $56.5\left(\mathrm{OCH}_{3}\right), 30.8$ $\left(\mathrm{CH}_{3}\right)$; EA (\%) calculated for $\mathrm{C}_{12} \mathrm{H}_{10} \mathrm{O}_{4}: 66.05 \mathrm{C}, 4.62 \mathrm{H}$; found: $66.15 \mathrm{C}, 4.60 \mathrm{H}$.

3-Acetyl-6,8-dichloro-2H-1-benzopyran-2-one (5g). Prepared from $0.5 \mathrm{~g}$ (2.6 mmol) of 3,5dichlorosalicylaldehyde and $0.33 \mathrm{~mL}(2.6 \mathrm{mmol})$ of ethyl acetoacetate. White solid in $48 \%$ yield, $\mathrm{mp}$ 172-175 ${ }^{\circ} \mathrm{C}$. IR v( $\left.\mathrm{cm}^{-1}\right)$ : $1749(\mathrm{OC}=\mathrm{O}), 1676(\mathrm{C}=\mathrm{O}), 1217(\mathrm{C}-\mathrm{O}), 769(\mathrm{C}-\mathrm{Cl}) .{ }^{1} \mathrm{H}-\mathrm{NMR}: 8.37$ (s, 1H, H4), $7.54\left(\mathrm{~d}, 1 \mathrm{H},{ }^{4} \mathrm{~J}=2.4, \mathrm{H} 7\right), 7.66\left(\mathrm{~d}, 1 \mathrm{H},{ }^{4} \mathrm{~J}=2.4, \mathrm{H} 5\right), 2.71\left(\mathrm{~s}, 3 \mathrm{H}, \mathrm{CH}_{3}\right) ;{ }^{13} \mathrm{C}-\mathrm{NMR} 194.8(\mathrm{CO})$, 157.7 (OCO), 149.7 (C10), 145.4 (C4), 122.9 (C8), 127.8 (C5), 126.2 (C3), 130.5 (C6), 134.2 (C7), 120.1 (C9), $30.7\left(\mathrm{CH}_{3}\right)$; EA (\%) calculated for $\mathrm{C}_{11} \mathrm{H}_{6} \mathrm{O}_{3} \mathrm{Cl}_{2}: 51.39 \mathrm{C}, 2.35 \mathrm{H}$; found: $51.53 \mathrm{C}, 2.42 \mathrm{H}$.

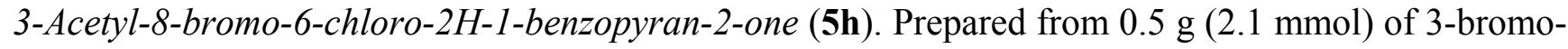
5-chloro-salicylaldehyde and $0.27 \mathrm{~mL}$ of ethyl acetoacetate $(2.1 \mathrm{mmol})$. Yellow solid in $60 \%$ yield, $\mathrm{mp}$ 192-196 ${ }^{\circ} \mathrm{C}$. IR v(cm $\left.{ }^{-1}\right): 1740(\mathrm{OC}=\mathrm{O}), 1675(\mathrm{C}=\mathrm{O}), 1202(\mathrm{C}-\mathrm{O}), 760(\mathrm{C}-\mathrm{Cl}), 556(\mathrm{C}-\mathrm{Br}) .{ }^{1} \mathrm{H}-\mathrm{NMR}$ (DMSO-d $\left.)_{6}\right): 8.55(\mathrm{~s}, 1 \mathrm{H}, \mathrm{H} 4), 8.05\left(\mathrm{~d}, 1 \mathrm{H},{ }^{4} \mathrm{~J}=2.2, \mathrm{H} 7\right), 8.12\left(\mathrm{~d}, 1 \mathrm{H},{ }^{4} \mathrm{~J}=2.2, \mathrm{H} 5\right), 2.56\left(\mathrm{~s}, 3 \mathrm{H}, \mathrm{CH}_{3}\right)$; ${ }^{13}$ C-NMR: 195.4 (CO), 158.0 (OCO), 150.7 (C10), 146.1 (C4), 110.5 (C8), 129.8 (C5), 126.6 (C3), 129.5 (C6), 136.5 (C7), 121.1 (C9), $30.7\left(\mathrm{CH}_{3}\right)$; EA (\%) calculated for $\mathrm{C}_{11} \mathrm{H}_{6} \mathrm{O}_{3} \mathrm{ClBr}$ : $43.82 \mathrm{C}, 2.01 \mathrm{H}$; found: $43.70 \mathrm{C}, 2.12 \mathrm{H}$.

3-Acetyl-6-bromo-8-methoxy-2H-1-benzopyran-2-one (5i). Prepared from $0.5 \mathrm{~g}$ (2.2 mmol) de 5-bromo-2-hydroxy-3-methoxybenzaldehyde and $0.28 \mathrm{~mL}$ of ethyl acetoacetate $(2.2 \mathrm{mmol})$. Yellow solid in 89\% yield, mp 215-218 ${ }^{\circ} \mathrm{C}$. IR $v\left(\mathrm{~cm}^{-1}\right): 1735(\mathrm{OC}=\mathrm{O}), 1674(\mathrm{C}=\mathrm{O}), 1235,1128(\mathrm{C}-\mathrm{O}), 662$ (C-Br). ${ }^{1} \mathrm{H}-\mathrm{NMR}: 8.36$ (s, 1H, H4), 7.24 (d, $\left.1 \mathrm{H},{ }^{4} \mathrm{~J}=2.1, \mathrm{H} 7\right), 7.34$ (d, $\left.1 \mathrm{H},{ }^{4} \mathrm{~J}=2.1, \mathrm{H} 5\right), 3.96$ (s, 3H, $\mathrm{OCH}_{3}$ ), 2.71 (s, 3H, $\left.\mathrm{CH}_{3}\right) ;{ }^{13} \mathrm{C}-\mathrm{NMR}: 195.1$ (CO), 158.0 (OCO), 144.0 (C10), 146.3 (C4), 147.6 (C8), 123.1 (C5), 125.5 (C3), 117.1 (C6), 118.8 (C7), 119.7 (C9), $56.5\left(\mathrm{OCH}_{3}\right), 30.5\left(\mathrm{CH}_{3}\right)$; EA (\%) calculated for $\mathrm{C}_{12} \mathrm{H}_{9} \mathrm{O}_{4} \mathrm{Br}$ : $48.5 \mathrm{C}, 3.1 \mathrm{H}$; found: $48.45 \mathrm{C}, 2.95$.

\subsection{General synthetic procedure for DA adducts 6-10}

$1 \mathrm{mmol}$ (typically 100-300 $\mathrm{mg}$ ) of the corresponding coumarin 1-5 and the appropriate volume equivalent to $6 \mathrm{mmol}$ of 2,3-dimethyl-1,3-butadiene (typically $0.3-1.0 \mathrm{~mL}$ ) were placed in a glass ampoule; the sealed ampoule was placed inside a metallic capsule and heated in a sand bath at $160{ }^{\circ} \mathrm{C}$ for a period of 24 hours. The ampoule content was dissolved in $\mathrm{CHCl}_{3}$ and evaporated to dryness. The resultant solid was washed with hot hexane and the insoluble solid was purified by $\mathrm{CC}$ in $\mathrm{SiO}_{2}-\mathrm{gel}$, using $\mathrm{CHCl}_{3}$ as eluent. Cycloadducts 6a [5], 7a [9] are reported elsewhere.

(6aR,10aR)- $\quad$ and (6aS,10aS)-N-[(R)-1-Phenylethyl]-6a,7,10,10a-tetrahydro-8,9-dimethyl-6-oxodibenzo[b,d]pyran-6a-carboxamide (8a). White crystalline solid in $77 \%$ yield, mp $168.9-171.4{ }^{\circ} \mathrm{C}$. 
IR/v $\left(\mathrm{cm}^{-1}\right): 3312(\mathrm{NH}), 1784(\mathrm{OC}=\mathrm{O}), 1629(\mathrm{NC}=\mathrm{O}), 1532(\mathrm{C}=\mathrm{C}), 1222(\mathrm{~N}-\mathrm{C}), 1146(\mathrm{C}-\mathrm{O}) .{ }^{1} \mathrm{H}-$ NMR: $7.30(\mathrm{~m}, 1 \mathrm{H}, \mathrm{H} 3), 7.26\left(\mathrm{t}, 2 \mathrm{H},{ }^{3} J=7.3\right.$ and $\left.7.7, \mathrm{Hm}\right), 7.13\left(\mathrm{t}, 1 \mathrm{H},{ }^{3} J=7.3, \mathrm{Hp}\right), 7.14(\mathrm{~d}, 2 \mathrm{H}$, $\left.{ }^{3} J=7.3, \mathrm{Ho}\right), 7.07\left(\mathrm{t}, 1 \mathrm{H},{ }^{3} J=8.1\right.$ and 8.4, H2), $7.00\left(\mathrm{~d}, 1 \mathrm{H},{ }^{3} J=8.1, \mathrm{H} 1\right), 6.83\left(\mathrm{dd}, 1 \mathrm{H},{ }^{3} J=5.3\right.$, $\left.{ }^{4} J=2.2, \mathrm{H} 4\right), 5.89,5.82\left(\mathrm{~d}, 1 \mathrm{H}\right.$ each, $\left.{ }^{3} J=7.5, \mathrm{~N}-\mathrm{H}\right), 4.88\left(\mathrm{dq}, 1 \mathrm{H},{ }^{3} J=7.3, \mathrm{CH}_{3}\right), 3.58(\mathrm{~m}, 1 \mathrm{H}, \mathrm{H} 10 \mathrm{a})$, $2.90\left(\mathrm{t}, 1 \mathrm{H},{ }^{3} \mathrm{~J}=18.3, \mathrm{H}_{\mathrm{eq}}-7\right), 2.49\left(\mathrm{~d}, 1 \mathrm{H},{ }^{3} \mathrm{~J}=17.4, \mathrm{H}_{\mathrm{ax}}-7\right), 2.32\left(\mathrm{t}, 1 \mathrm{H},{ }^{3} J=18.7\right.$ and $\left.12.3, \mathrm{H}_{\mathrm{eq}}-10\right)$, $2.02\left(\mathrm{~m}, 1 \mathrm{H}, \mathrm{H}_{\mathrm{ax}}-10\right), 1.67,1.60\left(\mathrm{~s}, 3 \mathrm{H}\right.$ each, $\left.2 \mathrm{CH}_{3}\right), 1.37,1.21\left(\mathrm{~d}, 3 \mathrm{H},{ }^{3} \mathrm{~J}=7.0, \mathrm{CH}_{3}\right) ;{ }^{13} \mathrm{C}-\mathrm{NMR}$ : 170.6, 170.5 (NCO), 167.6, 167.4 (OCO), 150.2 (C4a), 142.5, 142.2 (Ci), 129.0 (C3), 128.7, 128.6 $(\mathrm{Cm}), 128.5,128.4(\mathrm{C} 1), 128.1$ (C10b), 128.0, 127.7 (C2), 127.4, 126.1 (Cp), 125.5, 125.7 (Co), 123.5, 123.6 (C9), 123.2, 123.3 (C8), 116.8, 116.7 (C4), 55.0, 54.8 (C6a), 49.5, 49.2 (NCH), 37.0, 36.9 (C7), 36.8, $36.8(\mathrm{C} 10), 36.3(\mathrm{C} 10 \mathrm{a}), 21.6,21.4\left(\mathrm{CH}_{3} \mathrm{CH}\right), 18.8,18.8\left(\mathrm{CH}_{3}\right)$; GC/MS m/z (\%): $375\left(\mathrm{M}^{+}, 24\right)$, 281 (38), 227 (100), 211 (5), 199 (3), 105 (29), 79 (12), 44 (5).

(6aR,10aR)-rel-N-(2-Phenylethyl)-6a,7,10,10a-tetrahydro-8,9-dimethyl-6-oxodibenzo[b,d]pyran-6acarboxamide (9a). White crystalline powder in $84 \%$ yield, $\mathrm{mp} 138.6-140.0{ }^{\circ} \mathrm{C} . \mathrm{IR} / \mathrm{v}\left(\mathrm{cm}^{-1}\right): 3333$ $(\mathrm{NH}), 1771(\mathrm{OC}=\mathrm{O}), 1634(\mathrm{NC}=\mathrm{O}), 1546(\mathrm{C}=\mathrm{C}), 1222(\mathrm{C}-\mathrm{O}), 1144(\mathrm{C}-\mathrm{N}) .{ }^{1} \mathrm{H}-\mathrm{NMR}: 7.25$ (t, 2H, $\left.{ }^{3} J=7.3, \mathrm{Hm}\right), 7.27\left(\mathrm{~d}, 1 \mathrm{H},{ }^{3} J=7.3, \mathrm{H} 1\right), 7.21(\mathrm{dt}, 1 \mathrm{H}, 7.0 \mathrm{~Hz}, \mathrm{H} 3), 7.11\left(\mathrm{ddt}, 1 \mathrm{H},{ }^{3} J=7.2\right.$ and 7.7, $\left.{ }^{4} J=1.3, \mathrm{H} 2\right), 7.05\left(\mathrm{dd}, 2 \mathrm{H},{ }^{3} J=7.1, \mathrm{Ho}\right), 7.00\left(\mathrm{dd}, 1 \mathrm{H},{ }^{3} J=7.5, \mathrm{H} 4\right), 5.79\left(\mathrm{t}, 1 \mathrm{H},{ }^{3} J=5.4, \mathrm{NH}\right), 3.58$ (dd, $1 \mathrm{H},{ }^{3} J=11.7$ and 11.7, H10a), $3.34\left(\mathrm{dt}, 2 \mathrm{H},{ }^{3} \mathrm{~J}=7.2\right.$ and $\left.7.2,{ }^{4} \mathrm{~J}=2.0, \mathrm{NCH}_{2}\right), 2.79(\mathrm{~d}, 1 \mathrm{H}$, $\left.{ }^{3} J=17.1, \mathrm{H}_{\mathrm{eq}}-7\right), 2.61\left(\mathrm{~m}, 2 \mathrm{H}, \mathrm{CH}_{2}\right), 2.30\left(\mathrm{~d}, 1 \mathrm{H},{ }^{3} J=16.7, \mathrm{H}_{\mathrm{ax}}-7\right), 2.26\left(\mathrm{~d}, 1 \mathrm{H}, \mathrm{H}_{\mathrm{eq}}-10\right), 2.00(\mathrm{dd}, 1 \mathrm{H}$, ${ }^{3} J=15.5$ and $\left.12.0, \mathrm{H}_{\mathrm{ax}}-10\right), 1.58,1.65\left(\mathrm{~s}, 3 \mathrm{H}, 2 \mathrm{CH}_{3}\right) ;{ }^{13} \mathrm{C}-\mathrm{NMR}: 170.4(\mathrm{NC}=\mathrm{O}), 168.4(\mathrm{OC}=\mathrm{O}), 150.0$ (C4a), 138.4 (Ci), 129.0 (C10b), 128.9 (Co,m), 128.6 (Cp), 128.4 (C3), 127.9 (C1), 126.8 (C3), 125.4 (C2), 123.7 (C9), 123.1 (C8), 116.8 (C4), $41.3\left(\mathrm{NCH}_{2}\right), 37.3$ (C7), 36.5 (C10a), 36.3 (C13), 35.5 $\left(\mathrm{CH}_{2}\right)$, 18.8, $18.7\left(\mathrm{CH}_{3}\right)$; GC/MS m/z (\%): $375\left(\mathrm{M}^{+}, 20\right), 227$ (100), 199 (3), 173 (7); EA (\%) calculated for $\mathrm{C}_{24} \mathrm{H}_{25} \mathrm{O}_{3} \mathrm{~N}$ : $76.77 \mathrm{C}, 6.71 \mathrm{H}, 3.73 \mathrm{~N}$; found: $76.70 \mathrm{C}, 6.72 \mathrm{H}, 3.82 \mathrm{~N}$.

(6aR,10aR)-rel-6a-Acetyl-6a,7,10,10a-tetrahydro-8,9-dimethyl-6-oxodibenzo[b,d]pyran (10a). White crystalline powder in $83 \%$ yield, mp $86-88^{\circ} \mathrm{C}$. IR/v( $\left.\mathrm{cm}^{-1}\right)$ : $1763(\mathrm{OC}=\mathrm{O}), 1702(\mathrm{C}=\mathrm{O}), 1159,1140(\mathrm{C}$ O). ${ }^{1} \mathrm{H}-\mathrm{NMR}: 7.25(\mathrm{~m}, 1 \mathrm{H}, \mathrm{H} 3), 7.22\left(\mathrm{dd}, 1 \mathrm{H},{ }^{3} \mathrm{~J}=12.1,{ }^{4} \mathrm{~J}=1.8, \mathrm{H} 1\right), 7.10\left(\mathrm{td}, 1 \mathrm{H},{ }^{3} J=7.3\right.$, $\left.{ }^{4} J=1.2, \mathrm{H} 2\right), 7.03\left(\mathrm{dd}, 1 \mathrm{H},{ }^{3} J=7.9,{ }^{4} J=1.1, \mathrm{H} 4\right), 3.47\left(\mathrm{dd}, 1 \mathrm{H},{ }^{3} J=11.2\right.$ and 11.4, H10a), 2.87 (d, $\left.1 \mathrm{H},{ }^{3} J=17.3, \mathrm{H}_{\mathrm{eq}}-7\right), 2.31\left(\mathrm{dd}, 1 \mathrm{H},{ }^{3} J=18.0, \mathrm{H}_{\mathrm{ax}}-7\right), 2.24\left(\mathrm{~d}, 1 \mathrm{H},{ }^{3} J=12.0, \mathrm{H}_{\mathrm{eq}}-10\right), 2.12(\mathrm{~s}, 3 \mathrm{H}$, $\mathrm{CH}_{3}$ ), $2.06\left(\mathrm{dd}, 1 \mathrm{H}, \mathrm{H}_{\mathrm{ax}}-10\right), 1.70,1.62\left(\mathrm{~s}, 3 \mathrm{H}, \mathrm{CH}_{3}\right) ;{ }^{13} \mathrm{C}-\mathrm{NMR} 204.1$ (CO), 168.6 (OCO), 150.4 (C4a), 128.9 (C3), 127.6 (C1), 127.5 (C10b), 125.1 (C2), 123.5 (C9), 122.8 (C8), 117.2 (C4), 60.6 (C6a), 36.8 (C10a), 35.9 (C10), 34.8 (C7), 26.2 (C14), 18.8, $18.9\left(\mathrm{CH}_{3}\right) ; \mathrm{GC} / \mathrm{MS} \mathrm{m} / z(\%): 270\left(\mathrm{M}^{+}, 4\right)$, 227 (100), 199 (8), 185 (7), 173 (10), 43 (22); EA (\%) calculated for $\mathrm{C}_{17} \mathrm{H}_{18} \mathrm{O}_{3}: 75.53 \mathrm{C}, 6.73 \mathrm{H}$; found: $75.50,6.70 \mathrm{H}$.

(6aR,10aR)-rel-6a-Acetyl-2-chloro-6a,7,10,10a-tetrahydro-8,9-dimethyl-6-oxodibenzo[b,d]pyran (10b). Pale yellow solid in 50\% yield, mp 102-106 ${ }^{\circ} \mathrm{C}$. IR $/ v\left(\mathrm{~cm}^{-1}\right): 1781(\mathrm{OC}=\mathrm{O}), 1699(\mathrm{C}=\mathrm{O}), 1212$, 1139 (C-O), 814 (C-Cl). ${ }^{1} \mathrm{H}-\mathrm{NMR}: 7.27\left(\mathrm{dd}, 1 \mathrm{H},{ }^{3} \mathrm{~J}=8.0,{ }^{4} \mathrm{~J}=1.98, \mathrm{H} 3\right), 7.19\left(\mathrm{~d}, 1 \mathrm{H},{ }^{4} \mathrm{~J}=2.0, \mathrm{H} 1\right)$, $6.94\left(\mathrm{~d}, 1 \mathrm{H},{ }^{3} J=8.0 \mathrm{~Hz}, \mathrm{H} 4\right), 3.43\left(\mathrm{dd}, 1 \mathrm{H},{ }^{3} J=6.2,11.3, \mathrm{H} 10 \mathrm{a}\right), 2.86$ (d, $\left.1 \mathrm{H},{ }^{2} J=16.0, \mathrm{H}_{\mathrm{eq}}-7\right), 2.28$ (dd, $\left.1 \mathrm{H},{ }^{2} J=18.3,{ }^{3} J=6.2, \mathrm{H}_{\mathrm{eq}}-10\right), 2.13\left(\mathrm{~d}, 1 \mathrm{H},{ }^{2} J=16.0, \mathrm{H}_{\mathrm{ax}}-7\right), 2.11(\mathrm{~s}, 3 \mathrm{H}, \mathrm{H} 14), 2.02(\mathrm{dd}, 1 \mathrm{H}$, $\left.{ }^{2} J=18.3,{ }^{3} J=11.3, \mathrm{H}_{\mathrm{ax}}-10\right), 1.67\left(\mathrm{~s}, 3 \mathrm{H}, \mathrm{CH}_{3}\right), 1.59\left(\mathrm{~s}, 3 \mathrm{H}, \mathrm{CH}_{3}\right) ;{ }^{13} \mathrm{C}-\mathrm{NMR}: 203.5(\mathrm{CO}), 167.9$ 
(OCO), 148.9 (C4a), 130.1 (C10b), 129.3 (C2), 128.8 (C3), 127.6 (C1), 123.8 (C9), 122.9 (C8), 118.5 (C4), 60.2 (C6a), 36.6 (C10a), 35.7 (C10), 34.8 (C7), 26.3 (C14), 18.8, $18.9\left(\mathrm{CH}_{3}\right)$; GC/MS m/z (\%): $306\left(\mathrm{M}^{+}, 1\right), 261$ (100), 233 (11), 219 (11), 207 (10), 165 (5), 70 (7), 43 (26); EA (\%) calculated for $\mathrm{C}_{17} \mathrm{H}_{17} \mathrm{O}_{3} \mathrm{Cl}-0.4 \mathrm{H}_{2} \mathrm{O}$ : $65.45 \mathrm{C}, 5.75 \mathrm{H}$; found $65.58 \mathrm{C}, 5.79 \mathrm{H}$.

(6aR,10aR)-rel-6a-Acetyl-2-bromo-6a,7,10,10a-tetrahydro-8,9-dimethyl-6-oxodibenzo[b,d]pyran (10c). Pale yellow solid in 55\% yield, mp 50-54 ${ }^{\circ} \mathrm{C} . \mathrm{IR} / \mathrm{v}\left(\mathrm{cm}^{-1}\right): 1773(\mathrm{OC}=\mathrm{O}), 1711(\mathrm{C}=\mathrm{O}), 1205$, 1138 (C-O), 821 (C-Br). ${ }^{1} \mathrm{H}-\mathrm{NMR}: 7.30$ (dd, $\left.1 \mathrm{H},{ }^{3} \mathrm{~J}=7.2,{ }^{4} \mathrm{~J}=1.7, \mathrm{H} 3\right), 7.27$ (d, 1H, $\left.{ }^{4} \mathrm{~J}=1.7 \mathrm{~Hz}, \mathrm{H} 1\right)$, $6.86\left(\mathrm{~d}, 1 \mathrm{H},{ }^{3} J=7.2, \mathrm{H} 4\right), 3.41\left(\mathrm{dd}, 1 \mathrm{H},{ }^{3} J=6.4,11.4 \mathrm{~Hz}, \mathrm{H} 10 \mathrm{a}\right), 2.83\left(\mathrm{~d}, 1 \mathrm{H},{ }^{2} J=16.8, \mathrm{H}_{\mathrm{eq}}-7\right), 2.14$ (dd, $\left.1 \mathrm{H},{ }^{2} J=16.9,{ }^{3} J=11.4, \mathrm{H}_{\mathrm{eq}}-10\right), 2.09$ (s, 3H, H14), 2.04 (d, 1H, ${ }^{2} J=16.9, \mathrm{H}_{\mathrm{ax}}-7$ ), 1.92 (dd, 1H, $\left.{ }^{2} \mathrm{~J}=16.8,{ }^{3} \mathrm{~J}=6.4, \mathrm{H}_{\mathrm{ax}}-10\right), 1.57\left(\mathrm{~s}, 3 \mathrm{H}, \mathrm{CH}_{3}\right), 1.66\left(\mathrm{~s}, 3 \mathrm{H}, \mathrm{CH}_{3}\right) ;{ }^{13} \mathrm{C}-\mathrm{NMR}: 203.6$ (CO), 167.9 (OCO), 149.4 (C4a), 130.4 (C1), 131.8 (C3), 129.6 (C2), 123.4 (C9), 122.8 (C8), 118.9 (C4), 117.7 (C10b), 60.3 (C6a), 36.5 (C10a), 35.7 (C10), 34.7 (C7), 26.2 (C14), 18.8, $18.9\left(\mathrm{CH}_{3}\right) ; \mathrm{GC} / \mathrm{MS} \mathrm{m} / z(\%): 348$ $\left(\mathrm{M}^{+}, 1\right), 305$ (100), 277 (5), 251 (6), 198 (10), 165 (10), 43 (58); EA (\%) calculated for $\mathrm{C}_{17} \mathrm{H}_{17} \mathrm{O}_{3} \mathrm{Br}$ : C $58.47 \mathrm{C}, 4.91 \mathrm{H}$; found: C $58.43 \mathrm{C}, 4.82 \mathrm{H}$.

(6aR,10aR)-rel-6a-Acetyl-6a,7,10,10a-tetrahydro-8,9-dimethyl-2-nitro-6-oxodibenzo[b,d]pyran (10d). Pale yellow powder in 81\% yield, mp 142-147 C. IR/v( $\left.\mathrm{cm}^{-1}\right): 1782(\mathrm{OC}=\mathrm{O}), 1726(\mathrm{C}=\mathrm{O}), 1525$ $\left(\mathrm{NO}_{2}\right), 1339,1230(\mathrm{C}-\mathrm{O}) .{ }^{1} \mathrm{H}-\mathrm{NMR}: 8.16\left(\mathrm{~d}, 1 \mathrm{H},{ }^{4} \mathrm{~J}=2.5, \mathrm{H} 1\right), 8.14\left(\mathrm{dd}, 1 \mathrm{H},{ }^{3} J=9.3,{ }^{4} \mathrm{~J}=2.5, \mathrm{H} 3\right)$, $7.15\left(\mathrm{~d}, 1 \mathrm{H},{ }^{3} J=9.3, \mathrm{H} 4\right), 3.60\left(\mathrm{dd}, 1 \mathrm{H},{ }^{3} J=6.4,11.1, \mathrm{H} 10 \mathrm{a}\right), 2.90\left(\mathrm{~d}, 1 \mathrm{H},{ }^{2} J=17.3, \mathrm{H}_{\mathrm{eq}}-7\right), 2.38(\mathrm{~d}$, $1 \mathrm{H}$,

$\left.{ }^{2} J=17.3, \mathrm{H}_{\mathrm{ax}}-7\right), 2.27\left(\mathrm{dd}, 1 \mathrm{H},{ }^{2} J=16.7,{ }^{3} \mathrm{~J}=6.2, \mathrm{H}_{\mathrm{eq}}-10\right), 2.15$ (s, 3H, H14), 2.05 (dd, $1 \mathrm{H},{ }^{2} J=16.7$, $\left.{ }^{3} J=11.1, \mathrm{H}_{\mathrm{ax}}-10\right), 1.62\left(\mathrm{~s}, 3 \mathrm{H}, \mathrm{CH}_{3}\right), 1.71\left(\mathrm{~s}, 3 \mathrm{H}, \mathrm{CH}_{3}\right) ;{ }^{13} \mathrm{C}-\mathrm{NMR}: 202.7$ (CO), 166.9 (OCO), 154.7 (C4a), 144.6 (C2), 128.9 (C10b), 124.8 (C3), 123.6 (C1), 123.3 (C9), 122.9 (C8), 118.1 (C4), 60.1 (C6a), 36.5 (C10a), 35.6 (C10), $34.6(\mathrm{C} 7), 26.1$ (C14), 18.8, $18.9\left(\mathrm{CH}_{3}\right) ; \mathrm{GC} / \mathrm{MS}$ m/z (\%): $315\left(\mathrm{M}^{+}, 1\right)$, 272 (100), 244 (8), $230(8), 218(8), 204$ (6), 67 (7), 43 (25); EA (\%) calculated for $\mathrm{C}_{17} \mathrm{H}_{17} \mathrm{O}_{5} \mathrm{~N}: 64.75$ C, $5.43 \mathrm{H}, 4.44 \mathrm{~N}$; found $65.10 \mathrm{C}, 5.65 \mathrm{H}, 4.20 \mathrm{~N}$.

(6aR,10aR)-rel-6a-Acetyl-6a,7,10,10a-tetrahydro-2-methoxy-8,9-dimethyl-6-oxodibenzo[b,d]pyran (10e). Pale yellow solid in 55\% yield, m.p 44-46 ${ }^{\circ} \mathrm{C} . \mathrm{IR} / \mathrm{v}\left(\mathrm{cm}^{-1}\right): 1761(\mathrm{OC}=\mathrm{O}) 1710(\mathrm{C}=\mathrm{O})$ 1190, 1147 (C-O). NMR: ${ }^{1} \mathrm{H}-\mathrm{NMR}: 6.89\left(\mathrm{~d}, 1 \mathrm{H},{ }^{4} \mathrm{~J}=3.5, \mathrm{H} 1\right), 6.87\left(\mathrm{~d}, 1 \mathrm{H},{ }^{3} J=6.5, \mathrm{H} 4\right), 6.68$ (dd, $1 \mathrm{H}$, $\left.{ }^{3} J=6.5,{ }^{4} J=3.2, \mathrm{H} 3\right), 3.71\left(\mathrm{~s}, 3 \mathrm{H}, \mathrm{OCH}_{3}\right), 3.36\left(\mathrm{dd}, 1 \mathrm{H},{ }^{3} J=6.5,11.0, \mathrm{H} 10 \mathrm{a}\right), 2.78\left(\mathrm{~d}, 1 \mathrm{H},{ }^{2} J=16.7\right.$, $\left.\mathrm{H}_{\mathrm{eq}}-7\right), 2.19\left(\mathrm{dd}, 1 \mathrm{H},{ }^{2} J=18.2,{ }^{3} J=6.5, \mathrm{H}_{\mathrm{eq}}-10\right), 2.06$ (s, 3H, H14), 2.09 (d, 1H, $\left.{ }^{2} J=16.7, \mathrm{H}_{\mathrm{ax}}-7\right), 2.00$ (dd, $\left.1 \mathrm{H},{ }^{2} J=18.2,{ }^{3} J=11.0, \mathrm{H}_{\mathrm{ax}}-10\right), 1.59$ (s, 3H, $\left.\mathrm{CH}_{3}\right), 1.63\left(\mathrm{~s}, 3 \mathrm{H}, \mathrm{CH}_{3}\right) ;{ }^{13} \mathrm{C}-\mathrm{NMR}: 203.7$ (CO), 168.3 (OCO), 156.2 (C2), 143.8 (C4a), 128.0 (C3), 123.0 (C9), 122.4 (C8), 117.5 (C1), 113.2 (C10b), $112.3(\mathrm{C} 4), 60.0(\mathrm{C} 6 \mathrm{a}), 55.4\left(\mathrm{OCH}_{3}\right), 36.7$ (C10a), $35.4(\mathrm{C} 10), 34.4(\mathrm{C} 7), 25.9(\mathrm{C} 14), 18.4,18.5$ $\left(\mathrm{CH}_{3}\right)$; GC/MS m/z (\%): $300\left(\mathrm{M}^{+}, 1\right), 218(80), 190$ (5), 175 (15); EA (\%) calculated for $\mathrm{C}_{18} \mathrm{H}_{20} \mathrm{O}_{4}$ : $71.98 \mathrm{C}, 6.71 \mathrm{H}$; found: $71.91 \mathrm{C}, 6.65 \mathrm{H}$.

(6aR,10aR)-rel-6a-Acetyl-6a,7,10,10a-tetrahydro-4-methoxy-8,9-dimethyl-6-oxodibenzo[b,d]pyran (10f). Pale yellow powder $85 \%$ yield, mp 115-119 ${ }^{\circ} \mathrm{C}$. IR v( $\left.\mathrm{cm}^{-1}\right): 1769(\mathrm{OC}=\mathrm{O}), 1706(\mathrm{C}=\mathrm{O}), 1196$, 1095 (C-O). ${ }^{1} \mathrm{H}-\mathrm{NMR}: 7.03$ (dd, $\left.1 \mathrm{H},{ }^{3} J=8.4,7.7 \mathrm{H} 2\right), 6.82$ (d, $\left.1 \mathrm{H},{ }^{3} \mathrm{~J}=8.4, \mathrm{~Hz}, \mathrm{H} 1\right), 6.78(\mathrm{~d}, 1 \mathrm{H}$, 
$\left.{ }^{3} J=7.7, \mathrm{H} 3\right), 3.85\left(\mathrm{~s}, 3 \mathrm{H}, \mathrm{OCH}_{3}\right), 3.44\left(\mathrm{dd}, 1 \mathrm{H},{ }^{3} J=6.4,11.4, \mathrm{H} 10 \mathrm{a}\right), 2.88\left(\mathrm{~d}, 1 \mathrm{H},{ }^{2} J=16.7, \mathrm{H}_{\mathrm{eq}}{ }^{-7}\right)$, $2.21\left(\mathrm{dd}, 1 \mathrm{H},{ }^{2} \mathrm{~J}=18.2,{ }^{3} \mathrm{~J}=6.4, \mathrm{H}_{\mathrm{eq}}-10\right), 2.12(\mathrm{~s}, 3 \mathrm{H}, \mathrm{H} 14), 2.11\left(\mathrm{~d}, 1 \mathrm{H},{ }^{2} J=16.7, \mathrm{H}_{\mathrm{ax}}-7\right), 2.03$ (dd, $\left.1 \mathrm{H},{ }^{2} J=18.5,{ }^{3} \mathrm{~J}=11.4, \mathrm{H}_{\mathrm{ax}}-10\right), 1.68\left(\mathrm{~s}, 3 \mathrm{H}, \mathrm{CH}_{3}\right), 1.59$ (s, 3H, $\left.\mathrm{CH}_{3}\right) ;{ }^{13} \mathrm{C}-\mathrm{NMR}: 203.9$ (CO), 167.9 (OCO), 147.8 (C10), 139.9 (C8), 128.7 (C9), 125.2 (C6), 123.5 (C9), 122.8 (C8), 119.1 (C7), 111.6 (C5), $60.4(\mathrm{C6a}), 56.2\left(\mathrm{OCH}_{3}\right), 36.9(\mathrm{C} 10 \mathrm{a}), 35.8(\mathrm{C} 10), 34.9(\mathrm{C} 7), 26.4(\mathrm{C} 14), 18.8,18.9\left(\mathrm{CH}_{3}\right)$; GC/MS m/z (\%): $300\left(\mathrm{M}^{+}, 1\right), 257$ (100), 229 (7), 203 (8), 105 (2), 105 (2), 91 (4), 77 (4), 43 (17); EA (\%) calculated for $\mathrm{C}_{18} \mathrm{H}_{20} \mathrm{O}_{4}: 71.98 \mathrm{C}, 6.71 \mathrm{H}$; found: $72.25 \mathrm{C}, 6.80 \mathrm{H}$.

(6aR,10aR)-rel-6a-Acetyl-2,4-dichloro-6a,7,10,10a-tetrahydro-8,9-dimethyl-6-oxodibenzo[b,d]pyran (10g). Pale yellow powder in 71\% yield, mp $148-151{ }^{\circ} \mathrm{C} . \mathrm{IR} / \mathrm{v}\left(\mathrm{cm}^{-1}\right): 1787(\mathrm{OC}=\mathrm{O}), 1703(\mathrm{C}=\mathrm{O})$, 1233, 1192 (C-O), $810(\mathrm{C}-\mathrm{Cl}) .{ }^{1} \mathrm{H}-\mathrm{NMR}: 7.28$ (d, $\left.1 \mathrm{H},{ }^{4} \mathrm{~J}=2.4, \mathrm{H} 3\right), 7.11$ (d, $\left.1 \mathrm{H},{ }^{4} \mathrm{~J}=2.4, \mathrm{H} 1\right), 3.47$ (dd, $\left.1 \mathrm{H},{ }^{3} J=6.2,11.5, \mathrm{H} 10 \mathrm{a}\right), 2.89\left(\mathrm{~d}, 1 \mathrm{H},{ }^{2} J=17.0, \mathrm{H}_{\mathrm{eq}}-7\right), 2.28\left(\mathrm{dd}, 1 \mathrm{H},{ }^{2} J=17.9,{ }^{3} J=6.2, \mathrm{H}_{\mathrm{eq}}-10\right)$, $2.17\left(\mathrm{~d}, 1 \mathrm{H},{ }^{2} J=18.7, \mathrm{H}_{\mathrm{ax}}-7\right), 2.14$ (s, 3H, H14), 1.97 (dd, $\left.1 \mathrm{H},{ }^{2} J=18.9,{ }^{3} J=9.3, \mathrm{H}_{\mathrm{ax}}-10\right), 1.69$ (s, 3H, $\mathrm{CH}_{3}$ ), 1.59 (s, 3H, $\left.\mathrm{CH}_{3}\right) ;{ }^{13} \mathrm{C}-\mathrm{NMR}: 202.8(\mathrm{CO}), 166.8$ (OCO), 144.9 (C4a), 130.7 (C4), 130.1 (C2), 129.2 (C3), 126.1 (C1), 123.3 (C9), 122.9 (C8), 60.1 (C6a), 36.9 (C10a), 35.5 (C10), 34.9 (C7), 26.5 (C14), 18.8, $18.9\left(\mathrm{CH}_{3}\right)$; GC/MS m/z (\%): $339\left(\mathrm{M}^{+}, 1\right), 296$ (100), 268 (9), 253 (12), 241 (12), 232 (9), 91 (5), 67 (8), 43 (37); EA (\%) calculated for $\mathrm{C}_{17} \mathrm{H}_{16} \mathrm{O}_{3} \mathrm{Cl}_{2}: 60.19 \mathrm{C}, 4.75 \mathrm{H}$; found $60.15 \mathrm{C}, 4.85 \mathrm{H}$.

(6aR,10aR)-rel-6a-Acetyl-4-bromo-2-chloro-6a, 7, 10,10a-tetrahydro-8,9-dimethyl-6oxodibenzo[b,d]pyran (10h). Pale yellow powder in 62\% yield, $\mathrm{mp} 152-155^{\circ} \mathrm{C}$. IR v( $\left.\mathrm{cm}^{-1}\right): 1785$ $(\mathrm{OC}=\mathrm{O}), 1703(\mathrm{C}=\mathrm{O}), 1232,1131(\mathrm{C}-\mathrm{O}), 787(\mathrm{C}-\mathrm{Cl}), 524(\mathrm{C}-\mathrm{Br}) .{ }^{1} \mathrm{H}-\mathrm{NMR}: 7.45\left(\mathrm{~d}, 1 \mathrm{H},{ }^{4} \mathrm{~J}=2.4, \mathrm{H} 1\right)$, $7.15\left(\mathrm{~d}, 1 \mathrm{H},{ }^{4} \mathrm{~J}=2.4, \mathrm{H} 3\right), 3.46\left(\mathrm{dd}, 1 \mathrm{H},{ }^{3} \mathrm{~J}=6.4,11.4, \mathrm{H} 10 \mathrm{a}\right), 2.89\left(\mathrm{~d}, 1 \mathrm{H},{ }^{2} J=17.1, \mathrm{H}_{\mathrm{eq}}-7\right), 2.31(\mathrm{dd}$, $\left.1 \mathrm{H},{ }^{2} J=17.6,{ }^{3} J=6.4, \mathrm{H}_{\mathrm{eq}}-10\right), 2.19\left(\mathrm{~d}, 1 \mathrm{H},{ }^{2} J=16.9, \mathrm{H}_{\mathrm{ax}}-7\right), 2.14(\mathrm{~s}, 3 \mathrm{H}, \mathrm{H} 14), 1.99(\mathrm{dd}, 1 \mathrm{H}$, $\left.{ }^{2} J=17.6,{ }^{3} J=11.4, \mathrm{H}_{\mathrm{ax}}-10\right), 1.69\left(\mathrm{~s}, 3 \mathrm{H}, \mathrm{CH}_{3}\right), 1.60\left(\mathrm{~s}, 3 \mathrm{H}, \mathrm{CH}_{3}\right) ;{ }^{13} \mathrm{C}-\mathrm{NMR}: 202.8(\mathrm{CO}), 166.9$ (OCO), 146.1 (C4a), 132.0 (C3), 130.6 (C2), 130.4 (C4), 126.8 (C1), 123.3 (C9), 122.9 (C8), 111.4 (C10b), $60.1(\mathrm{C} 6 \mathrm{a}), 37.0(\mathrm{C} 10 \mathrm{a}), 35.5(\mathrm{C} 10), 34.9(\mathrm{C} 7), 26.6(\mathrm{C} 14), 18.8,18.9\left(\mathrm{CH}_{3}\right)$; GC/MS m/z (\%): $384\left(\mathrm{M}^{+}, 1\right), 341$ (100), 325 (5), 313 (6), 287 (10), 232 (15), 67 (16), 43 (27); EA (\%) calculated for $\mathrm{C}_{17} \mathrm{H}_{16} \mathrm{O}_{3} \mathrm{ClBr}$ : $53.22 \mathrm{C}, 4.2 \mathrm{H}$; found $53.60 \mathrm{C}, 4.25 \mathrm{H}$.

(6aR,10aR)-rel-6a-Acetyl-2-bromo-6a, 7, 10,10a-tetrahydro-4-methoxy-8,9-dimethyl-6-oxodibenzo[b,d]pyran (10i). Pale yellow powder in 83\% yield, mp 160-164 ${ }^{\circ} \mathrm{C} . \mathrm{IR} / \mathrm{v}\left(\mathrm{cm}^{-1}\right): 1769(\mathrm{OC}=\mathrm{O}), 1706$ $(\mathrm{C}=\mathrm{O}), 1275,1196$ (C-O), 506 (C-Br). ${ }^{1} \mathrm{H}-\mathrm{NMR}: 6.94$ (s, 1H, H1), 6.94 (s, 1H, H3), 3.84 (s, 3H, $\left.\mathrm{OCH}_{3}\right), 3.41\left(\mathrm{dd}, 1 \mathrm{H},{ }^{3} J=6.2,11.3, \mathrm{H} 10 \mathrm{a}\right), 2.88\left(\mathrm{~d}, 1 \mathrm{H},{ }^{2} J=16.9, \mathrm{H}_{\mathrm{eq}}-7\right), 2.36(\mathrm{dd}, 1 \mathrm{H}$, $\left.{ }^{2} J=17.0,{ }^{3} J=6.2, \mathrm{H}_{\mathrm{eq}}-10\right), 2.18\left(\mathrm{~d}, 1 \mathrm{H},{ }^{2} J=16.9, \mathrm{H}_{\mathrm{ax}}-7\right), 2.13(\mathrm{~s}, 3 \mathrm{H}, \mathrm{H} 14), 1.97\left(\mathrm{dd}, 1 \mathrm{H},{ }^{2} J=17.0\right.$, $\left.{ }^{3} J=11.3, \mathrm{H}_{\mathrm{ax}}-10\right), 1.68\left(\mathrm{~s}, 3 \mathrm{H}, \mathrm{CH}_{3}\right), 1.59\left(\mathrm{~s}, 3 \mathrm{H}, \mathrm{CH}_{3}\right) ;{ }^{13} \mathrm{C}-\mathrm{NMR}: 203.4(\mathrm{C}=\mathrm{O}), 167.4(\mathrm{OCO}), 148.4$ (C4a), 138.5 (C4), 130.3 (C2), 123.4 (C9), 122.8 (C8), 121.9 (C3), 117.6 (C10b), 114.9 (C1), 60.2 (C6a), 56.5 (s, 3H, $\left.\mathrm{OCH}_{3}\right), 36.7$ (C10a), 35.6 (C10), 34.9 (C7), 26.4 (C14), 18.8, $18.8\left(\mathrm{CH}_{3}\right)$; GC/MS m/z (\%): $380\left(\mathrm{M}^{+}, 1\right), 337$ (100), 309 (4), 283 (8), 256 (8), 228 (14), 115 (5), 91 (5), 43 (31); EA (\%) calculated for $\mathrm{C}_{18} \mathrm{H}_{19} \mathrm{O}_{4} \mathrm{Br}$ : 57.01 C, $5.05 \mathrm{H}$; found $57.20 \mathrm{C}, 5.06 \mathrm{H}$. 


\subsection{General synthetic procedure for epoxides 11-15}

Prepared from $200 \mathrm{mg}$ of compounds 6-10 and two equivalents of $\mathrm{m}$-CPBA dissolved in $\mathrm{CHCl}_{3}$ $(25 \mathrm{~mL})$ and refluxed for $24 \mathrm{~h}$. The $\mathrm{CHCl}_{3}$ solution was extracted with an aqueous saturated $\mathrm{NaHCO}_{3}$ solution. The organic layer was dried with $\mathrm{Na}_{2} \mathrm{SO}_{4}$, then filtered and concentrated.

Ethyl (6aR, 7aR,8aS,9aR)-rel-6a,7,7a,8a,9,9a-hexahydro-7a,8a-dimethyl-6-oxo-5,8-dioxacyclopropa[b]phenantrene-6a(6H)-carboxylate (11a). White crystalline solid in $73 \%$ yield, mp $79.8-82.5{ }^{\circ} \mathrm{C}$. $\mathrm{IR} / \mathrm{v}\left(\mathrm{cm}^{-1}\right): 1772(\mathrm{OC}=\mathrm{O}), 1735(\mathrm{EtOC}=\mathrm{O}), 1248,1230,1150(\mathrm{C}-\mathrm{O}) .{ }^{1} \mathrm{H}-\mathrm{NMR}: 7.19$ (ddd, 1H, $\left.{ }^{3} J=7.7,7.7,{ }^{4} J=1.8 \mathrm{~Hz}, \mathrm{H} 3\right), 7.09\left(\mathrm{dd}, 1 \mathrm{H},{ }^{3} J=7.5,{ }^{4} \mathrm{~J}=1.8, \mathrm{H} 1\right), 7.03$ (dd, $1 \mathrm{H},{ }^{3} J=7.5,{ }^{4} J=1.1$, $\mathrm{H} 2), 6.98\left(\mathrm{~d}, 1 \mathrm{H},{ }^{3} J=8.6, \mathrm{H} 4\right), 3.87\left(\mathrm{~m}, 2 \mathrm{H}, \mathrm{OCH}_{2}\right), 3.36\left(\mathrm{dd}, 1 \mathrm{H},{ }^{3} J=12.4\right.$ and 5.5, H9a), 2.83 (d, $\left.1 \mathrm{H},{ }^{3} J=15.2, \mathrm{H}_{\mathrm{eq}}-7\right), 2.24\left(\mathrm{~d}, 1 \mathrm{H},{ }^{3} J=15.4, \mathrm{H}_{\mathrm{ax}}-7\right), 2.21\left(\mathrm{dd}, 1 \mathrm{H},{ }^{3} J=10.3\right.$ and $\left.5.5, \mathrm{H}_{\mathrm{eq}}-9\right), 1.60$ (dd, $1 \mathrm{H},{ }^{3} J=15.6$ and 12.5, $\left.\mathrm{H}_{\mathrm{ax}}-9\right), 1.40,1.24\left(\mathrm{~s}, 3 \mathrm{H} \mathrm{c} / \mathrm{u}, 2 \mathrm{CH}_{3}\right), 0.83\left(\mathrm{t}, 3 \mathrm{H}, \mathrm{CH}_{3}-\mathrm{CH}_{2}\right) .{ }^{13} \mathrm{C}-\mathrm{NMR}: 169.9$ (OCO), 167.4 (OCO lactone), 150.9 (C4a), 129.1 (C1), 127.8 (C3), 126.4 (C9b), 125.1 (C2), 117.0 (C4), $62.3\left(\mathrm{CH}_{2}-\mathrm{O}\right), 61.7$ (C8a), 60.3 (C7a, 53.3 (C6a), 35.6 (C9a), 34.5 (C9), $34.4(\mathrm{C} 7), 21.0\left(\mathrm{CH}_{3^{-}}\right.$ C8a), $19.2\left(\mathrm{CH}_{3}-\mathrm{C} 7 \mathrm{a}\right), 13.9\left(\mathrm{CH}_{3}-\mathrm{CH}_{2}\right)$; GC/MS m/z (\%): $316\left(\mathrm{M}^{+}, 2\right), 259$ (38), 243 (100), 225 (50), 214 (34), 199 (24), 145 (11), 115 (17), 91 (6), 43 (52); EA (\%) calculated for $\mathrm{C}_{18} \mathrm{H}_{20} \mathrm{O}_{5}$ : $68.34 \mathrm{C}, 6.37$ $\mathrm{H}$; found: $68.35 \mathrm{C}, 6.32 \mathrm{H}$.

(6aR,7aR,8aS,9aR)-rel-N-benzyl-6a, 7,7a,8a,9,9a-hexahydro-7a,8a-dimethyl-6-oxo-5,8-dioxacyclopropa[b]phenantrene-6a(6H)-carboxamide (12a). White crystalline solid in $78 \%$ yield, mp $205.4-207{ }^{\circ} \mathrm{C}$. $\mathrm{IR} / v\left(\mathrm{~cm}^{-1}\right): 3342(\mathrm{NH}), 1773(\mathrm{OC}=\mathrm{O}), 1637(\mathrm{NC}=\mathrm{O}), 1538(\mathrm{C}=\mathrm{C}), 1234(\mathrm{C}-\mathrm{O}), 1152(\mathrm{C}-\mathrm{N}) .{ }^{1} \mathrm{H}-$ NMR: $7.27(\mathrm{~m}, 1 \mathrm{H}, \mathrm{H} 3), 7.21\left(\mathrm{~m}, 5 \mathrm{H}, 5 \mathrm{H}-\mathrm{C}_{6} \mathrm{H}_{5}\right), 7.12\left(\mathrm{t}, 1 \mathrm{H},{ }^{3} J=7.4, \mathrm{H} 6\right), 7.01\left(\mathrm{~d}, 1 \mathrm{H},{ }^{3} J=8.0, \mathrm{H} 5\right)$, $6.82(\mathrm{~m}, 1 \mathrm{H}, \mathrm{H} 4), 5.82(\mathrm{t}, 1 \mathrm{H}, \mathrm{NH}), 4.22\left(\mathrm{~m}, 2 \mathrm{H}, \mathrm{AA}{ }^{\prime} \mathrm{BB}\right.$ ', $\left.\mathrm{NCH}_{2}\right), 3.52\left(\mathrm{dd}, 1 \mathrm{H},{ }^{2} J=12.5,{ }^{3} J=5.3\right.$, H9a), $2.94\left(\mathrm{~d}, 1 \mathrm{H},{ }^{2} J=15.5, \mathrm{H}_{\mathrm{eq}}-7\right), 2.32\left(\mathrm{dd}, 1 \mathrm{H},{ }^{2} J=15.5,{ }^{3} J=5.2, \mathrm{H}_{\mathrm{eq}}-9\right), 2.27\left(\mathrm{~d}, 1 \mathrm{H},{ }^{2} J=15.5\right.$, $\left.\mathrm{H}_{\mathrm{ax}}-7\right), 1.69\left(\mathrm{dd}, 1 \mathrm{H},{ }^{2} J=15.6,{ }^{3} J=12.7, \mathrm{H}_{\mathrm{ax}}-9\right), 1.43\left(\mathrm{~s}, 3 \mathrm{H}, \mathrm{CH}_{3}\right), 1.30\left(\mathrm{~s}, 3 \mathrm{H}, \mathrm{CH}_{3}\right) ;{ }^{13} \mathrm{C}-\mathrm{NMR}$ : 169.4 (NCO), 168.0 (OCO lactone), 150.3 (C4a), 137.4 (Ci), 128.9 (C-m and C1), 128.0 (C3), 127.8 $(\mathrm{Cp}), 127.5(\mathrm{Co}), 127.0(\mathrm{C} 9 \mathrm{~b}), 125.6(\mathrm{C} 2), 117.0(\mathrm{C} 4), 61.8$ (C8a), 60.5 (7a), $54.6(\mathrm{C} 6 \mathrm{a}), 43.9\left(\mathrm{NCH}_{2}\right)$, 36.0 (C9a), 35.7 (C9), 34.7 (C8a), $21.0\left(\mathrm{CH}_{3}-\mathrm{C} 8 \mathrm{a}\right), 19.1\left(\mathrm{CH}_{3}-\mathrm{C} 7 \mathrm{a}\right)$; GC/MS m/z (\%): $377\left(\mathrm{M}^{+}, 2\right), 318$ (10), 243 (40), 227 (15), 225 (30), 211 (32), 199 (8), 173 (8), 150 (100), 131 (10), 91 (95), 43 (30); EA (\%) calculated for $\mathrm{C}_{23} \mathrm{H}_{23} \mathrm{O}_{4} \mathrm{~N}$ : $73.19 \mathrm{C}, 6.14 \mathrm{H}$; found: $73.09 \mathrm{C}, 6.14 \mathrm{H}$.

(6aR,7aR,8aS,9aR)- and (6aS,7aS,8aR,9aS)-6a,7,7a,8a,9,9a-hexahydro-7a,8a-dimethyl-6-oxo-N-[(R)1-phenylethyl]-5,8-dioxacyclopropa[b]phenantrene-6a(6H)-carboxamide (13a). White crystalline powder in 78\% yield, mp 163.9-165.0 ${ }^{\circ} \mathrm{C}$; IR $\vee\left(\mathrm{cm}^{-1}\right)$ : $3256(\mathrm{NH}), 1759(\mathrm{OC}=\mathrm{O}), 1638(\mathrm{NC}=\mathrm{O})$, 1230, 1161, 1151 (C-O); GC/MS m/z (\%) $391\left(\mathrm{M}^{+}, 4\right), 332$ (15), 243 (58), 227 (28), 225 (35), 211 (42), 199 (8), 173 (15), 105 (100), 43 (20). Minor (6aR,7aR,8aS,9aR) isomer 13a: ${ }^{1} \mathrm{H}-\mathrm{NMR}: 7.3-6.9$ (m, 9H, ArH), $5.67\left(\mathrm{~d}, 1 \mathrm{H},{ }^{3} J=1.5, \mathrm{NH}\right), 3.48\left(\mathrm{dd}, 1 \mathrm{H},{ }^{3} J=5.1,{ }^{3} J=12.0, \mathrm{H} 9 \mathrm{a}\right), 2.92\left(\mathrm{dd}, 1 \mathrm{H},{ }^{2} J=\right.$ 15.4, $\left.\mathrm{H}_{\mathrm{eq}}-7\right), 2.27\left(\mathrm{~d}, 1 \mathrm{H},{ }^{2} J=15.6, \mathrm{H}_{\mathrm{ax}}-7\right), 2.29\left(\mathrm{~m}, 1 \mathrm{H}, \mathrm{H}_{\mathrm{eq}}-9\right), 1.68\left(\mathrm{dd}, 1 \mathrm{H},{ }^{2} J=15.6,{ }^{3} J=5.6, \mathrm{H}_{\mathrm{ax}}{ }^{-}\right.$ 9), $4.84\left(\mathrm{dq}, 1 \mathrm{H},{ }^{3} \mathrm{~J}=6.9, \mathrm{CHCH}_{3}\right), 1.43$ and $1.30\left(\right.$ two s, $2 \times 3 \mathrm{H}, 7 \mathrm{a}-$ and $\left.8 \mathrm{a}-\mathrm{CH}_{3}\right), 1.26\left(\mathrm{~d}, 3 \mathrm{H},{ }^{3} J=\right.$ 7.0, $\left.\mathrm{CHCH}_{3}\right)$; ${ }^{13} \mathrm{C}-\mathrm{NMR}: 169.6$ (NCO), 167.1 (C-6), 150.3 (C-4a), 142.0 (C-1'), 128.8 (C-3',5'), 128.0 (C-1), no (C-3), 127.6 (C-9b), 127.0 (C-4'), 125.9 (C-2',6’), 125.5 (C-2), 117.0 (C-4), 61.8 (C-8a), 
60.6 (C-7a), 54.6 (C-6a), 49.1 (NCH), 35.9 (C-9a), 34.9 (C-9), 34.8 (C-7), 21.1, 21.0, and 19.1 $\left(3 \times \mathrm{CH}_{3}\right)$. Major (6aS,7aS,8aR,9aS) isomer 13a: ${ }^{1} \mathrm{H}-\mathrm{NMR}: 7.3-6.9(\mathrm{~m}, 9 \mathrm{H}, \mathrm{ArH}), 5.71\left(\mathrm{~d}, 1 \mathrm{H},{ }^{3} J=\right.$ $1.5, \mathrm{NH}), 3.44\left(\mathrm{dd}, 1 \mathrm{H},{ }^{3} J=5.1,{ }^{3} J=12.0, \mathrm{H}-9 \mathrm{a}\right), 2.90\left(\mathrm{dd}, 1 \mathrm{H},{ }^{2} J=15.4, \mathrm{H}_{\mathrm{eq}}-7\right), 2.19$ (d, $1 \mathrm{H},{ }^{2} J=$ 15.6, $\left.\mathrm{H}_{\mathrm{ax}}-7\right), 2.29\left(\mathrm{~m}, 1 \mathrm{H}, \mathrm{H}_{\mathrm{eq}}-9\right), 1.68\left(\mathrm{dd}, 1 \mathrm{H},{ }^{2} J=15.6,{ }^{3} J=5.6, \mathrm{H}_{\mathrm{ax}}-9\right), 4.83\left(\mathrm{dq}, 1 \mathrm{H},{ }^{3} J=6.9\right.$, $\mathrm{CHCH}_{3}$ ), 1.42 and 1.29 (two s, $2 \times 3 \mathrm{H}, 7 \mathrm{a}-$ and $\left.8 \mathrm{a}-\mathrm{CH}_{3}\right), 1.22\left(\mathrm{~d}, 3 \mathrm{H},{ }^{3} \mathrm{~J}=7.0, \mathrm{CHCH}_{3}\right) ;{ }^{13} \mathrm{C}-\mathrm{NMR}$ : 169.3 (NCO), 167.1 (C-6), 150.4 (C-4a), 142.1 (C-1'), 129.0 (C-3',5'), no (C-1), 127.9 (C-3), 127.7 (C-9b), 126.8 (C-4'), 126.1 (C-2',6'), 125.5 (C-2), 117.0 (C-4), 61.8 (C-8a), 60.6 (C-7a), 54.5 (C-6a), $49.2(\mathrm{NCH}), 36.0$ (C-9a), $35.5(\mathrm{C}-9), 34.8(\mathrm{C}-7), 21.1,21.0$, and $19.1\left(3 \times \mathrm{CH}_{3}\right)$.

(6aR,7aR,8aS,9aR)-rel-6a, 7, 7a,8a,9,9a-Hexahydro-7a,8a-dimethyl-6-oxo-N-(2-phenylethyl)-5,8dioxacyclopropa[b]phenantrene-6a(6H)-carboxamide (14a). White crystalline powder in $78 \%$ yield, mp 189.4-191.6 ${ }^{\circ} \mathrm{C}$. IR/v (cm $\left.{ }^{-1}\right)$ : $3322(\mathrm{NH}), 1789$ (OC=O), 1640 (CONH), 1146 y 1129 (C-O). ${ }^{1} \mathrm{H}$ - NMR: 7.25(m, 5H, Ph), $7.15\left(\mathrm{dd}, 1 \mathrm{H},{ }^{3} \mathrm{~J}=7.4,{ }^{4} \mathrm{~J}=1.9, \mathrm{H} 3\right), 7.10\left(\mathrm{dd}, 1 \mathrm{H},{ }^{3} \mathrm{~J}=7.4,{ }^{4} \mathrm{~J}=1.2, \mathrm{H} 2\right)$, $7.03\left(\mathrm{~d}, 1 \mathrm{H},{ }^{3} \mathrm{~J}=6.7, \mathrm{H} 1\right), 7.00\left(\mathrm{~d}, 1 \mathrm{H},{ }^{3} \mathrm{~J}=8.1, \mathrm{H} 4\right), 5.54(\mathrm{a}, 1 \mathrm{H}, \mathrm{NH}), 3.48\left(\mathrm{dd}, 1 \mathrm{H},{ }^{3} J=5.2\right.$, $\left.{ }^{3} J=12.6, \mathrm{H} 9 \mathrm{a}\right), 3.29$ (q, $\left.2 \mathrm{H},{ }^{3} J=7.2, \mathrm{NCH}_{2}\right), 2.81$ (d, $\left.1 \mathrm{H},{ }^{3} J=5.5, \mathrm{H}_{\mathrm{eq}}-7\right), 2.56\left(\mathrm{t}, 2 \mathrm{H},{ }^{3} J=7.2, \mathrm{CH}_{2}\right.$ ), $2.28\left(\mathrm{dd}, 1 \mathrm{H},{ }^{3} J=5.2,{ }^{2} J=15.7, \mathrm{H}_{\mathrm{eq}}-9\right), 2.11\left(\mathrm{~d}, 1 \mathrm{H},{ }^{3} J=15.7, \mathrm{H}_{\mathrm{ax}}-7\right), 1.65\left(\mathrm{dd}, 1 \mathrm{H},{ }^{3} J=5.5 \mathrm{~Hz}\right.$,

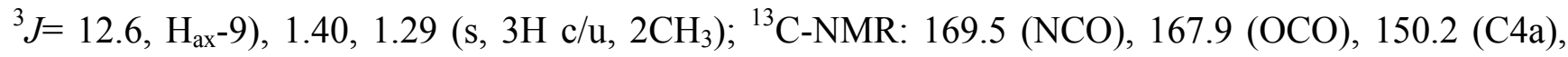
$138.3(\mathrm{C} i), 128.9(\mathrm{Cm}), 128.9$ (Co), 128.8 (C1), 127.9 (C3), 126.9 (Cp), 125.7 (C2), 116.9 (C4), 127.1 (C9b), 61.8 (C8a), 60.5 (C7a), 54.4 (C6a), $41.2\left(\mathrm{NCH}_{2}\right), 36.0\left(\mathrm{CH}_{2}\right), 35.9(\mathrm{C} 9 \mathrm{a}), 35.4(\mathrm{C} 9), 34.5(\mathrm{C} 7)$, $21.0\left(\mathrm{CH}_{3} \mathrm{C} 8 \mathrm{a}\right), 19.1\left(\mathrm{CH}_{3} \mathrm{C} 7 \mathrm{a}\right) ; \mathrm{GC} / \mathrm{MS} \mathrm{m} / z(\%) 391\left(\mathrm{M}^{+}, 8\right), 332$ (20), 243 (100), 227 (43), 225 (95), 211 (68), 173 (43); EA (\%) calculated for $\mathrm{C}_{24} \mathrm{H}_{25} \mathrm{O}_{4} \mathrm{~N}$ : $73.64 \mathrm{C}, 6.44 \mathrm{H}, 3.58 \mathrm{~N}$; found: $73.27 \mathrm{C}, 6.24$ $\mathrm{H}, 3.44 \mathrm{~N}$.

(6aR, 7aR,8aS, 9aR)-rel-6a-Acetyl-6a,7,7a,8a,9,9a-hexahydro-7a,8a-dimethyl-6-oxo-6H-5,8dioxacyclopropa[b]phenantrene (15a). White crystalline powder in $70 \%$ yield, mp $125.6-126.9{ }^{\circ} \mathrm{C}$. $\mathrm{IR} / v\left(\mathrm{~cm}^{-1}\right): 1772(\mathrm{OC}=\mathrm{O}), 1699(\mathrm{C}=\mathrm{O}), 1300,1258,1150(\mathrm{C}-\mathrm{O}) .{ }^{1} \mathrm{H}-\mathrm{NMR}: 7.25\left(\mathrm{dd},{ }^{1} \mathrm{H},{ }^{3} J=7.6,7.4\right.$, H2), $7.16\left(\mathrm{~d},{ }^{3} J=7.6, \mathrm{H} 1\right), 7.09\left(\mathrm{dd}, 1 \mathrm{H},{ }^{3} J=7.4,8.1, \mathrm{H} 3\right), 7.02$ (d, $\left.1 \mathrm{H},{ }^{3} J=8.1, \mathrm{H} 4\right), 3.45$ (dd, $1 \mathrm{H}$, $\left.{ }^{3} J=12.2,5.0, \mathrm{H} 9 \mathrm{a}\right), 2.83\left(\mathrm{~d}, 1 \mathrm{H},{ }^{2} J=15.0, \mathrm{H}_{\mathrm{eq}}-7\right), 2.31\left(\mathrm{dd}, 1 \mathrm{H},{ }^{2} J=15.4,{ }^{3} J=5.0, \mathrm{H}_{\mathrm{eq}}-9\right), 2.08(\mathrm{~d}$, $\left.1 \mathrm{H},{ }^{2} J=15.0, \mathrm{H}_{\mathrm{ax}}-7\right), 2.02\left(\mathrm{~s}, 3 \mathrm{H}, \mathrm{CH}_{3} \mathrm{O}\right), 1.68\left(\mathrm{dd}, 1 \mathrm{H},{ }^{2} J=15.4,{ }^{3} J=12.2, \mathrm{H}_{\mathrm{ax}}-9\right), 1.45,1.31(\mathrm{~s}, 3 \mathrm{H}$ c/u, $\left.2 \mathrm{CH}_{3}\right)$; ${ }^{13} \mathrm{C}-\mathrm{NMR}: 203.5\left(\mathrm{COCH}_{3}\right), 170.4(\mathrm{OCO}), 150.6(\mathrm{C} 4 \mathrm{a}), 134.9(\mathrm{C} 9 \mathrm{~b}), 130.5(\mathrm{C} 1), 128.5$ (C2), 125.4 (C3), 117.4 (C4), 62.0 (C8a), 60.4 (C7a), 60.3 (C6a), 35.9 (C9a), 34.3 (C9), 33.2 (C7), $25.5\left(\mathrm{CH}_{3} \mathrm{CO}\right), 21.0\left(\mathrm{CH}_{3} \mathrm{C} 8 \mathrm{a}\right), 19.2\left(\mathrm{CH}_{3} \mathrm{C} 7 \mathrm{a}\right)$; GC/MS m/z (\%): $286\left(\mathrm{M}^{+}, 1\right), 243(16), 225(28), 211$ (100), 184 (14), 114 (17), 91 (9), 43 (60); EA (\%) calculated for $\mathrm{C}_{17} \mathrm{H}_{18} \mathrm{O}_{4}$ : $71.31 \mathrm{C}, 6.34 \mathrm{H}$; found: $71.28 \mathrm{C}, 6.44 \mathrm{H}$.

(6aR,7aR,8aS,9aR)-rel-6a-Acetyl-6a,7,7a,8a,9,9a-hexahydro-7a,8a-dimethyl-2-nitro-6-oxo-6H-5,8dioxacyclopropa[b]phenantrene (15d). White solid in $96 \%$ yield, $\mathrm{mp} 216-220{ }^{\circ} \mathrm{C} . \mathrm{IR} / \mathrm{v}\left(\mathrm{cm}^{-1}\right): 1786$ $(\mathrm{OC}=\mathrm{O}), 1700(\mathrm{C}=\mathrm{O}), 1526\left(\mathrm{NO}_{2}\right), 1338,1237,1142(\mathrm{C}-\mathrm{O}) .{ }^{1} \mathrm{H}-\mathrm{NMR}: 8.18\left(\mathrm{dd}, 1 \mathrm{H},{ }^{4} \mathrm{~J}=2.6,{ }^{3} \mathrm{~J}=8.8\right.$, H3), 8.15 (d, $\left.1 \mathrm{H},{ }^{4} J=2.6, \mathrm{H} 1\right), 7.18\left(\mathrm{~d}, 1 \mathrm{H},{ }^{3} J=8.8, \mathrm{H} 4\right), 3.63$ (dd, $\left.1 \mathrm{H},{ }^{3} J=5.2,12.1, \mathrm{H} 9 \mathrm{a}\right), 2.89$ (d, $\left.1 \mathrm{H},{ }^{2} J=15.5, \mathrm{H}_{\mathrm{eq}} 7\right), 2.38\left(\mathrm{dd}, 1 \mathrm{H},{ }^{2} J=15.5,{ }^{3} J=5.2, \mathrm{H}_{\mathrm{eq}}-9\right), 2.15\left(\mathrm{~d}, 1 \mathrm{H},{ }^{2} J=15.5, \mathrm{H}_{\mathrm{ax}}-7\right), 2.09$ (s, $3 \mathrm{H}, \mathrm{H} 14), 1.70\left(\mathrm{dd}, 1 \mathrm{H},{ }^{2} \mathrm{~J}=15.5,{ }^{3} \mathrm{~J}=12.1, \mathrm{H}_{\mathrm{ax}}-9\right), 1.35\left(\mathrm{~s}, 3 \mathrm{H}, \mathrm{CH}_{3}\right), 1.48\left(\mathrm{~s}, 3 \mathrm{H}, \mathrm{CH}_{3}\right)$; ${ }^{13} \mathrm{C}-\mathrm{NMR}$ : 202.2 (CO), 166.8 (OCO), 154.8 (C4a), 144.7 (C2), 127.4 (C9b), 125.2 (C3), 123.6 (C1), 118.2 (C4), 
61.6 (C6a), 60.0 (C8a), 59.9 (C7a), 35.6 (C9), 34.2 (C9a), 32.9 (C7), 25.4 (C14), 20.9, $19.0\left(\mathrm{CH}_{3}\right)$; GC/MS m/z (\%): $331\left(\mathrm{M}^{+}, 1\right), 288$ (19), 256 (100), 230 (26), 115 (5), 91 (4), 43 (78); EA (\%) calculated for $\mathrm{C}_{17} \mathrm{H}_{17} \mathrm{O}_{6} \mathrm{~N}$ : $61.63 \mathrm{C}, 5.17 \mathrm{H}, 4.23 \mathrm{~N}$; found: $61.44 \mathrm{C}, 5.25 \mathrm{H}, 4.14 \mathrm{~N}$.

(6aR,7aR,8aS,9aR)-rel-6a-Acetyl-6a, 7,7a,8a,9,9a-hexahydro-4-methoxy-7a,8a-dimethyl-6-oxo-6H5,8-dioxacyclopropa[b]phenantrene (15f). White solid in $92 \%$ yield, mp $188-192{ }^{\circ} \mathrm{C} . \mathrm{IR} / \mathrm{v}\left(\mathrm{cm}^{-1}\right)$ : $1764(\mathrm{OC}=\mathrm{O}), 1702(\mathrm{C}=\mathrm{O}), 1281,1153,1095(\mathrm{C}-\mathrm{O}) .{ }^{1} \mathrm{H}-\mathrm{NMR}: 7.05$ (t, 1H, H2, $\left.{ }^{3} \mathrm{~J}=7.9\right), 6.85$ (dd, $1 \mathrm{H}$,

$\left.{ }^{3} J=7.9, \mathrm{H} 1\right), 6.74\left(\mathrm{dd}, 1 \mathrm{H},{ }^{3} J=7.9, \mathrm{H} 3\right), 3.85\left(\mathrm{~s}, 3 \mathrm{H}, \mathrm{OCH}_{3}\right), 3.44\left(\mathrm{dd}, 1 \mathrm{H},{ }^{3} J=5.0,12.3, \mathrm{H} 9 \mathrm{a}\right), 2.85$ $\left(\mathrm{d}, 1 \mathrm{H},{ }^{2} J=15.2, \mathrm{H}_{\mathrm{eq}}-7\right), 2.31\left(\mathrm{dd}, 1 \mathrm{H},{ }^{2} J=15.5,{ }^{3} J=5.0, \mathrm{H}_{\mathrm{eq}}-9\right), 2.07\left(\mathrm{~d}, 1 \mathrm{H},{ }^{2} J=15.2, \mathrm{H}_{\mathrm{ax}}-7\right), 2.04$ (s, 3H, H14), $1.70\left(\mathrm{dd}, 1 \mathrm{H},{ }^{2} J=15.5,{ }^{3} J=12.3, \mathrm{H}_{\mathrm{ax}}-9\right), 1.45\left(\mathrm{~s}, 3 \mathrm{H}, \mathrm{CH}_{3}\right), 1.31\left(\mathrm{~s}, 3 \mathrm{H}, \mathrm{CH}_{3}\right) ;{ }^{13} \mathrm{C}-$ NMR: 203.4 (CO), 167.8 (OCO), 147.8 (C4a), 139.6 (C4), 127.2 (C9b), 125.5 (C2), 118.9 (C1), 111.9 (C3), 61.9 (C6a), $60.3(\mathrm{C} 8 \mathrm{a}), 60.1(\mathrm{C} 7 \mathrm{a}), 56.3\left(\mathrm{OCH}_{3}\right), 35.6(\mathrm{C} 9), 34.4(\mathrm{C} 9 \mathrm{a}), 33.2(\mathrm{C} 7), 25.6(\mathrm{C} 14)$, 19.2, $21.0\left(\mathrm{CH}_{3}\right)$; GC/MS m/z (\%): $316\left(\mathrm{M}^{+}, 1\right), 273$ (53), 255 (100), 245(4), 241 (60), 211 (11), 115 (13), 91 (8), 43 (47); EA (\%) calculated for $\mathrm{C}_{18} \mathrm{H}_{20} \mathrm{O}_{5}$ : $68.34 \mathrm{C}, 6.37 \mathrm{H}$; found: $64.83 \mathrm{C}, 6.28 \mathrm{H}$.

(6aR,7aR,8aS,9aR)-rel-6a-Acetyl-2-bromo-6a, 7,7a,8a,9,9a-hexahydro-4-methoxy-7a,8a-dimethyl-6oxo-6H-5,8-dioxacyclopropa[b]phenantrene (15i). White powder in 95\% yield, mp $169-172{ }^{\circ} \mathrm{C}$. $\mathrm{IR} / \mathrm{v}\left(\mathrm{cm}^{-1}\right): 1768(\mathrm{OC}=\mathrm{O}), 1702(\mathrm{C}=\mathrm{O}), 1198,1154,1123(\mathrm{C}-\mathrm{O}), 512(\mathrm{C}-\mathrm{Br}) .{ }^{1} \mathrm{H}-\mathrm{NMR}: 6.97(\mathrm{~d}, 1 \mathrm{H}$, $\left.{ }^{4} J=2.1, \mathrm{H} 1\right), 6.91\left(\mathrm{~d}, 1 \mathrm{H},{ }^{4} J=2.1, \mathrm{H} 3\right), 3.86\left(\mathrm{~s}, 3 \mathrm{H}, \mathrm{OCH}_{3}\right), 3.42\left(\mathrm{dd}, 1 \mathrm{H},{ }^{3} J=5.0,12.3, \mathrm{H} 9 \mathrm{a}\right), 2.85$ $\left(\mathrm{d}, 1 \mathrm{H},{ }^{2} J=15.5, \mathrm{H}_{\mathrm{eq}}-7\right), 2.30\left(\mathrm{dd}, 1 \mathrm{H},{ }^{2} J=15.5,{ }^{3} J=5.0, \mathrm{H}_{\mathrm{eq}}-9\right), 2.07(\mathrm{~s}, 3 \mathrm{H}, \mathrm{H} 14), 2.06(\mathrm{~d}, 1 \mathrm{H}$, $\left.{ }^{2} J=15.5, \mathrm{H}_{\mathrm{ax}}-7\right), 1.68\left(\mathrm{dd}, 1 \mathrm{H},{ }^{2} J=15.5,{ }^{3} \mathrm{~J}=12.3, \mathrm{H}_{\mathrm{ax}}-9\right), 1.45\left(\mathrm{~s}, 3 \mathrm{H}, \mathrm{CH}_{3}\right), 1.32\left(\mathrm{~s}, 3 \mathrm{H}, \mathrm{CH}_{3}\right) ;{ }^{13} \mathrm{C}-$ NMR: 202.8 (CO), 167.2 (OCO), 148.5 (C4a), 138.7 (C4), 128.8 (C2), 121.7 (C3), 117.8 (C9b), 115.4 (C1), 61.7 (C8a), 60.2 (C7a), 59.9 (C6a), $56.4\left(\mathrm{OCH}_{3}\right), 35.4$ (C9), 34.3 (C9a), 33.2 (C7), 25.7 (C14), 20.9, $19.2\left(\mathrm{CH}_{3}\right)$; GC/MS m/z (\%): $395\left(\mathrm{M}^{+}, 1\right), 351$ (27), 334 (10), 321 (100), 255 (8), 240 (28), 115 (12), 91 (5), 43 (80); EA (\%) calculated for $\mathrm{C}_{18} \mathrm{H}_{19} \mathrm{O}_{5} \mathrm{Br}$ : $54.70 \mathrm{C}, 4.85 \mathrm{H}$; found: $54.26 \mathrm{C}, 4.74 \mathrm{H}$.

\subsection{Crystal structures}

Compound 10b: $\mathrm{C}_{17} \mathrm{H}_{17} \mathrm{C} \mathrm{O}$, colorless crystals, monoclinic, P21/c, Z = 4, $a=18.809(2) \AA$, $b=7.1461(9) \AA, c=11.4261(14) \AA, \alpha=90^{\circ}, \beta=105.493(2)^{\circ}, \gamma=90^{\circ}, V=1480.0(3) \AA^{3}$, $\mathrm{D}_{\text {calcd }}=1.368 \mathrm{~g} / \mathrm{cm}^{3}, \mu=0.265 \mathrm{~mm}^{-1}, 10086$ reflections collected, 2603 independent $\left(\mathrm{R}_{\text {int }}=0.025\right)$, 2414 observed, $\mathrm{R} 1=0.0411, w \mathrm{R} 2=0.1198(I>2 \sigma(I))$.

Compound 15i: $\mathrm{C}_{18} \mathrm{H}_{19} \mathrm{BrO}_{5}$, colorless crystals, triclinic, $P-1, \mathrm{Z}=2, a=7.3676(18) \AA$, $b=10.851(3) \AA, c=12.154(3) \AA, \alpha=108.055(4)^{\mathrm{o}}, \beta=97.784(4)^{\mathrm{o}}, \gamma=106.789(4)^{\mathrm{o}}, \mathrm{V}=857.0(4) \AA^{3}$, $\mathrm{D}_{\text {calcd }}=1.532 \mathrm{~g} / \mathrm{cm}^{3}, \mu=2.423 \mathrm{~mm}^{-1}, 8373$ reflections collected, 3017 independent $\left(\mathrm{R}_{\text {int }}=0.046\right), 2455$ observed, $\mathrm{R} 1=0.0575, w \mathrm{R} 2=0.1149(I>2 \sigma(I))$. 


\section{Conclusions}

The thermal reactions of ethyl coumarin-3-carboxylate (1a), 3-carboxyamides $\mathbf{2 a - 4 a}$ and 3-acetylcoumarins 5a-i with 2,3-dimethyl-1,3-butadiene under SFC yielded the corresponding DA adducts in 60 to $85 \%$, as a racemic mixture of the cis fused rings. Poor asymmetric induction is observed when the enantiopure compound 3a was used. Epoxidation of DA cycloadducts 6a-10a proceeded in $70-80 \%$ yield, whereas starting from $10 \mathrm{~d}$, 10f, and $10 \mathbf{i}$, the isolated yields were in the 90-96\% range. NMR and X-ray data demonstrated that the oxygen atom is stereoselectively added to the less hindered face of the cyclohexene ring, opposite to the benzopyrone ring fusion. ${ }^{1} \mathrm{H}-\mathrm{NMR}$ and X-ray data supported an anchored twisted boat conformation for both dihydropyrone and cyclohexene rings. Data on the supramolecular structure of DA adducts and epoxides is scarce, although it is directed by $\mathrm{CH} \cdots \mathrm{A}(\mathrm{A}=\mathrm{O}, \pi)$ and, in the case of $\mathbf{1 5 i}$, also by $\mathrm{Br} \cdots \mathrm{Br}$ interactions.

\section{Acknowledgements}

This work was supported by SIP-IPN (Secretaría de Investigación y Postgrado del Instituto Politécnico Nacional), CGIC-UC (Coordinación General de Investigación Científica de la Universidad de Colima), and CONACYT-83378. Thanks to UAEH (Universidad Autónoma del Estado de Hidalgo) for the access to the elemental analyzer.

\section{References and Notes}

1. Mustafa, A.; Kamel, M.; Allam, M.A. Dimerization reactions in eunlight. V. Photodimerization of substituted coumarins. J. Org. Chem. 1957, 22, 888-891.

2. Ayala-Hurtado, S.; Flores-Larios, I.Y.; Padilla-Martínez, I.I.; Martínez-Martínez, F.J.; GarcíaBáez, E.V.; Cruz, A.; Höpfl, H. From solid state photodimers of ethyl coumarin-3-carboxylate to their alcoholysis derivatives. A supramolecular study. Supramol. Chem. 2007, 19, 629-640.

3. Ohkata, K.; Lee, Y.-G.; Utsumi, Y.; Ishimaru, K.; Akiba, K.-Y. Diels-Alder reactions of 2[(trialkylsilyl)oxy]pyrylium cations of $2 \mathrm{H}$-pyran-2-one and $2 \mathrm{H}$-1-benzopyran-2-one derivatives. J. Org. Chem. 1991, 56, 5052-5059.

4. Amantini, D.; Fringuelli, F.; Pizzo, F. Uncatalyzed [4 +2] cycloadditions of 3-nitrocoumarins with vinyl ethers in solventless conditions. A new entry to chromene derivatives. J. Org. Chem. 2002, 67, 7238-7243.

5. Girotti, R.; Marrocchi, A.; Minuti, L.; Piermatti, O.; Pizzo, F.; Vaccaro, L. Diels-Alder reactions of 3-substituted coumarins in water and under high-pressure condition. An uncatalyzed route to tetrahydro-6H-benzo[c]chromen-6-ones. J. Org. Chem. 2006, 71, 70-74.

6. Ballerini, E.; Minuti, L.; Piermatti, O.; Pizzo, F. High pressure Diels-Alder approach to hydroxysubstituted 6a-cyano-tetrahydro-6H-benzo[c]chromen-6-ones: A route to $\Delta^{6}$-cis-cannabidiol. $J$. Org. Chem. 2009, 74, 4311-4317.

7. Fringuelli, F.; Girotti, R.; Pizzo, F.; Zunino, E.; Vaccaro, L. Hafnium chloride tetrahydrofuran complex-catalyzed Diels-Alder cycloadditions of 3-ethoxycarbonylcoumarins with 1,3-dienes under solvent-free Conditions. Adv. Synth. Catal. 2006, 348, 297-300. 
8. López-Garrido, L.; García-Báez, E.V.; Martínez-Martínez, F.J.; Padilla-Martínez I.I. 3-Acyl coumarin derivatives. $[2+2]$ and $[2+4]$ cycloaddition reactions. In proceeding of XXVI LatinAmerican Congress of Chemistry and 27 Annual Meeting of the Brazilian Chemical Society, Salvador Bahia, Brazil, May 30-June 2, 2004; Abstract QO-039.

9. Gotthardt, H.; Hoffmann, N. Synthese und neue $[4+2]$-Cycloadditionen von CumarinAbkömmlingen. Liebigs Ann. Chem. 1985, 901-912.

10. Ohkata, K.; Miyamoto, K.; Matsumura, S.; Akiba, K.-Y. Highly asymmetric induction in the Diels-Alder reaction of 3-alkoxycarbonyl-substituted coumarin. Tetrahedron Lett. 1993, 34, 6575-6578.

11. Martínez-Martínez, F.J.; Padilla-Martínez, I.I.; Trujillo-Ferrara, J. ${ }^{1} \mathrm{H}$ and ${ }^{13} \mathrm{C}$ NMR assignments of 2-oxo-2H-1-benzopyran-3-acyl and 3-amide derivatives. Magn. Res. Chem. 2001, 39, 765-767.

12. Munshi, P.; Venugopala, K.N.; Jayashree, B.S.; Guru-Row, T.N. Concomitant polymorphism in 3-acetylcoumarin: Role of weak $\mathrm{C}-\mathrm{H} \cdots \mathrm{O}$ and $\mathrm{C}-\mathrm{H} \cdots \pi$ interactions. Cryst. Growth Des. 2004, 4, 1105-1107.

13. Santos-Contreras, R.J.; Martínez-Martínez, F.J.; Mancilla-Margalli, N.A.; Peraza-Campos, A.L.; Morín-Sánchez, L.M.; García-Báez, E.V.; Padilla-Martínez, I.I. Competition between OH $\cdots \mathrm{O}$ and multiple halogen-dipole interactions on the formation of intramolecular three centred hydrogen bond in 3-acyl coumarins. CrystEngComm 2009, 11, 1451-1461.

14. Desiraju, G.R. The $\mathrm{C}-\mathrm{H} \cdots \mathrm{O}$ hydrogen bond: structural implications and supramolecular design. Acc. Chem. Res. 1996, 29, 441-449.

15. Umezawa, Y.; Tsuboyama, S.; Honda, K.; Uzawa, J.; Nishio, M. CH/ $\pi$ interaction in the crystal structure of organic compounds. A database study. Bull. Chem. Soc. Jpn. 1998, 71, 1207-1213.

16. García-Báez, E.V.; Martínez-Martínez, F.J.; Höpfl, H.; Padilla-Martínez, I.I. $\pi$-Stacking interactions and $\mathrm{C}-\mathrm{H} \cdots \mathrm{X}(\mathrm{X}=\mathrm{O}$, aryl $)$ hydrogen bonding as directing features of the supramolecular self-association in 3-carboxy and 3-amido coumarin derivatives. Cryst. Growth Des. 2003, 3, 35-45.

17. El-Deen, I.M.; Abd El-Fattah, M.; Ibrahim, H.K.; El-Gohary, A. Synthesis and investigation of mass spectra of 3-[5'-(2'-substituent)thienyl]benzo[5,6]coumarins. Phosphor. Sulfur. Silicon 2003, 178, 1463-1476.

18. Bruker. APEX II, SAINT, SADABS and SHELXTL. Bruker AXS Inc.: Madison, WI, USA, 2004.

19. Sheldrick, G.M. A short history of SHELX. Acta Crystallogr. A 2008, A64, 112-122.

20. Farrugia, L.J. WinGX suite for small-molecule single-crystal crystallography. J. Appl. Crystallogr. 1999, 32, 837-838.

21. Macrae, C.F.; Edgington, P.R.; McCabe, P.; Pidcock, E.; Shields, G.P.; Taylor, R.; Towler, M.; van de Streek, J. Mercury: Visualization and analysis of crystal structures. J. Appl. Crystallogr. 2006, 39, 453-457.

22. Spek, A.L. Single-crystal structure validation with the program PLATON. J. Appl. Crystallogr. 2003, 36, 7-13. 
23. Frisch, M.J.; Trucks, G.W.; Schlegel, H.B.; Scuseria, G.E.; Robb, M.A.; Cheeseman, J.R.; Zakrzewski, V.G.; Montgomery J.A.; Stratmann, R.E., Jr.; Burant, J.C.; Dapprich, S.; Millam, J.M.; Daniels, A.D.; Kudin, K.N.; Strain, M.C.; Farkas, O.; Tomasi, J.; Barone, V.; Cossi, M.; Cammi, R.; Mennucci, B.; Pomelli, C.; Adamo, C.; Clifford, S.; Ochterski, J.; Peterson, G.A.; Ayala, P.Y.; Cui, Q.; Morokuma, K.; Malick, D.K.; Rabuck, A.D.; Raghavachari, K.; Foresman, J.B.; Cioslowski, J.; Ortiz, J.V.; Baboul, A.G.; Stefanov, B.B.; Liu, G.; Liashenko, A.; Piskorz, P.; Komaromi, I.; Gomperts, R.; Martin, R.L.; Fox, D.J.; Keith, T.; Al-Laham, M.A.; Peng, C.Y.; Nanayakkara, A.; Challacombe, M.; Gill, P.M.W.; Johnson, B.; Chen, W.; Wong, M.W.; Andres, J.L.; Gonzalez, C.; Head-Gordon, M.; Replogle, E.S.; Pople, J.A. Gaussian 98, Revision A.9; Gaussian, Inc.: Pittsburgh, PA, USA, 1998.

24. Bonsignore, L.; Cottiglia, F.; Maccioni, A.M.; Secci, D.; Lavagna, S.M. Synthesis of coumarin-3$O$-acylisoureas by dicyclohexylcarbodiimide. J. Heterocycl. Chem. 1995, 32, 573-577.

25. Bonsignore, L.; Cottiglia, F.; Maccioni, A.M.; Secci, D.; Lavagna, S.M. Synthesis of coumarin-3$O$-acylisoureas by dicyclohexylcarbodiimide. J. Heterocycl. Chem. 1995, 32, 573-577.

Sample Availability: Samples of compounds 10a, 11-12a and 15a are available from the authors.

(C) 2010 by the authors; licensee Molecular Diversity Preservation International, Basel, Switzerland. This article is an open-access article distributed under the terms and conditions of the Creative Commons Attribution license (http://creativecommons.org/licenses/by/3.0/). 